\title{
General equilibrium and the new neoclassical synthesis
}

Citation for published version (APA):

Herings, P. J. J. (2012). General equilibrium and the new neoclassical synthesis. METEOR, Maastricht University School of Business and Economics. METEOR Research Memorandum No. 045 https://doi.org/10.26481/umamet.2012045

Document status and date:

Published: 01/01/2012

DOI:

10.26481/umamet.2012045

Document Version:

Publisher's PDF, also known as Version of record

\section{Please check the document version of this publication:}

- A submitted manuscript is the version of the article upon submission and before peer-review. There can be important differences between the submitted version and the official published version of record.

People interested in the research are advised to contact the author for the final version of the publication, or visit the DOI to the publisher's website.

- The final author version and the galley proof are versions of the publication after peer review.

- The final published version features the final layout of the paper including the volume, issue and page numbers.

Link to publication

\footnotetext{
General rights rights.

- You may freely distribute the URL identifying the publication in the public portal. please follow below link for the End User Agreement:

www.umlib.nl/taverne-license

Take down policy

If you believe that this document breaches copyright please contact us at:

repository@maastrichtuniversity.nl

providing details and we will investigate your claim.
}

Copyright and moral rights for the publications made accessible in the public portal are retained by the authors and/or other copyright owners and it is a condition of accessing publications that users recognise and abide by the legal requirements associated with these

- Users may download and print one copy of any publication from the public portal for the purpose of private study or research.

- You may not further distribute the material or use it for any profit-making activity or commercial gain

If the publication is distributed under the terms of Article $25 \mathrm{fa}$ of the Dutch Copyright Act, indicated by the "Taverne" license above, 


\section{Maastricht University}

P. Jean-J acques Herings

General Equilibrium and The New Neoclassical Synthesis

$\mathrm{RM} / 12 / 045$

\section{METEOR}

Maastricht University School of Business and Economics

Maastricht Research School of Economics

of Technology and Organization

P.O. Box 616

NL - 6200 MD Maastricht

The Netherlands 


\title{
General Equilibrium and the New Neoclassical Synthesis
}

\author{
P. Jean-Jacques Herings*
}

September 6, 2012

\begin{abstract}
We present a general equilibrium model of the new neoclassical synthesis that has the same level of generality as the Arrow-Debreu model. This involves a stochastic multiperiod economy with a monetary sector and sticky commodity prices. We formulate the notion of a sticky price equilibrium where all agents form rational expectations on prices for commodities and assets, interest rates, and rationing. We present a general result showing that monetary policy imposes no restrictions whatsoever on nominal equilibrium price levels and that the set of sticky price equilibria has a dimension equal to the number of terminal date-events. Stickiness of prices implies that this indeterminacy is real.
\end{abstract}

KEYWORDS: general equilibrium, monetary policy, sticky prices, new neoclassical synthesis, indeterminacy.

JEL CODES: D50, D90, E40, E50.

${ }^{*}$ P.J.J. Herings, Department of Economics, Maastricht University, P.O. Box 616, 6200 MD, Maastricht, The Netherlands. The author would like to thank Jacques Drèze for many helpful discussions on this subject. He would also like to thank the Netherlands Organization for Scientific Research (NWO) for financial support. P. Herings@maastrichtuniversity.nl 


\section{Introduction}

The new neoclassical synthesis is a term used by Goodfriend and King (1997) to refer to a class of models that incorporate elements of apparently irreconcilable traditions of macroeconomic thought. On the one hand there are the flexible price models of the new classical macroeconomists and real-business-cycle analysis, in which monetary policy is unimportant for real economic activity, and on the other hand there are the sticky-price models of the New Keynesian economics, in which monetary policy is central to understanding real economic activity. The integration of these two streams in the literature leads to a class of models with four central elements: intertemporal optimization, rational expectations, optimal price-setting, and costly price adjustment.

The workhorse of the new neoclassical synthesis is a simple general equilibrium model involving inflation, output, and nominal interest rates at various date-events as its main variables. Crucial in these models is the existence of imperfections in setting commodity prices, which causes them to respond with some lag to changes in market conditions. Price stickiness is then the main channel through which monetary policy affects real variables. The main objective is to study how aggregate variables like inflation and output are affected by the adoption of various alternative policies.

The aim of the current paper is to provide a formulation of the new neoclassical synthesis that has the same level of generality as the Arrow-Debreu general equilibrium model. This involves an extension of the new neoclassical workhorse to a setting with multiple commodities, multiple heterogeneous agents having general preferences, general monetary transaction technologies, and a general approach towards price stickiness. In such an extension, households optimize intertemporally given rationally anticipated prices of commodities and assets, interest rates, and the monetary transactions technology, and whenever prices are not sticky, they do not adjust mechanically to some measure of disequilibrium, but are derived endogenously.

To achieve these objectives, we extend the Arrow-Debreu model in three ways. First, we follow Arrow (1953) and specify a setting with sequentially opening markets for commodities as opposed to the Arrow-Debreu framework with a complete set of markets for contingent commodities. Second, we follow Clower (1967) and require that all purchases and sales of commodities in spot markets are made against money. We specify a general monetary transaction technology in the spirit of Drèze and Polemarchakis (2001). Third, we introduce a general model of price stickiness where future commodity prices are either inherited from the past or are determined endogenously to achieve market clearing. Our modeling of price stickiness incorporates frequently used specifications by Taylor (1980) and Calvo (1983) as special cases.

A particular period in the model starts with transactions on the asset markets with 
households trading Arrow securities and collecting dividends on their previous period's asset portfolios. Next, during the period, transactions on spot markets for commodities take place against money, and transactions with the bank occur exchanging bank loans against money. Monetary needs are determined by the monetary transactions technology and depend on commodity prices and the consumption bundles that are chosen. The bank charges interest on nominal debts, which households pay at the end of the period. Since households hold nominal debts in the aggregate, the bank creates seignorage, which is returned to the bank's shareholders at the end of the period in the form of dividends. This modeling choice implies that we implicitly assume a Ricardian fiscal policy.

The policy of the bank involves the specification of interest rates that are set conditional on the date-event. This is in accordance with the observation in Woodford (2003) that monetary policy decision making by central banks almost everywhere means a decision about the operating target for an overnight interest rate. The reason for making the interest rate the operating target is that the alternative where the bank tries to directly control monetary aggregates has become less effective as a consequence of increases in the sophistication of the financial system.

A central feature of the new neoclassical synthesis is that monetary policy has nontrivial consequences. The key reason is the assumption that commodity prices are not continually adjusted, but remain fixed for at least short periods. Indeed, price stickiness is a well-documented empirical phenomenon. Nakamura and Steinsson (2010), for instance, report that the median duration of a price across sectors is around one year. Price stickiness can be caused by a variety of reasons, but the typical explanation follows Sheshinksi and Weiss (1977) and is based on the existence of menu costs, caused both by the real costs associated with the transmission of prices to the consumers as well as with the decision process itself.

The standard approach in the macroeconomic literature is to specify constant elasticity of substitution in utility and production functions à la Dixit and Stiglitz (1977). This makes it possible to derive closed-form expressions for prices that are set by producers in an environment of imperfect competition. Such an approach does not generalize to a setting with general preferences as has been argued by Roberts and Sonnenschein (1977), who show that equilibrium price and quantity choices may fail to exist even in extremely simple cases. To keep the feature that prices are determined endogenously in equilibrium, we stick to the standard general equilibrium paradigm of competitive markets. At all date-events where the price of a commodity can be adjusted, its level is determined by the forces of supply and demand, where supply and demand is derived from fully rational intertemporally optimizing agents.

In periods where commodity prices are fixed, prices are typically not market clearing, 
resulting in excess supply or excess demand of commodities. In periods where prices cannot adjust, markets are equilibrated by quantity adjustments, where the long side of the market is rationed by the amount of trade on the short side. Here we follow the modeling approach developed by Drèze (1975) in the context of a general equilibrium model with upper and lower bounds on prices.

We formulate the concept of a sticky price equilibrium. At a sticky price equilibrium all households optimize given rational expectations. More precisely, at a sticky price equilibrium all households hold common and correct point expectations of all prices, rationing, interest rates, and dividends conditional on all possible date-events. Prices and rationing schemes are determined endogenously by the requirement of market clearing on commodity markets and asset markets, where sticky prices are set equal to the previous period's value. Allocations of commodities, assets, and money follow from optimizing behavior by the households, subject to the constraints imposed by the monetary transaction technology.

Our goal is to demonstrate the existence of a sticky price equilibrium under general assumptions on initial endowments, preferences, transaction technologies, and price stickiness. In an ad hoc macroeconomic model, Sargent and Wallace (1975) developed the insight that the price level is indeterminate under an interest rate rule. This insight has spurred an extensive literature debating the indeterminacy of equilibrium in models with Ricardian fiscal policy, see Woodford (2003) for a detailed treatment of this literature and Cochrane (2011) for a recent discussion. Beyond equilibrium existence, we are therefore interested in obtaining an indeterminacy of equilibrium result in our general setting.

For each terminal date-event, we select one commodity with a flexible price, and set this price equal to an arbitrary value. Next we prove that each such specification of prices is consistent with some sticky price equilibrium, which demonstrates that sticky price equilibria exist and that the set of sticky price equilibria has dimension at least equal to the number of terminal date-events. The equilibrium nominal price level at terminal dateevents is arbitrary, irrespective of the interest rate policy by the bank. We argue that price stickiness implies that this indeterminacy is real.

Our model contains several widely studied general equilibrium models as special cases. This brings up the issue as to how the indeterminacy result is related to equilibrium existence results that have appeared previously in the literature. The standard ArrowDebreu model corresponds to the case with one time period, one terminal date-event, no price stickiness, and zero interest rates charged by the bank on nominal debt. Such a model has a one-dimensional multiplicity of equilibrium indeed, which is usually suppressed by making use of zero-homogeneity of demand functions to normalize prices. Indeterminacy of equilibrium is entirely nominal.

The standard model of price rigidities as presented in Drèze (1975) corresponds to the 
case with one time period, one terminal date-event, and zero interest rates charged by the bank on nominal debt. For this model one-dimensional multiplicity of equilibrium is shown in Herings (1996a), extending such a result for supply-constrained equilibria in van der Laan (1982). For a one-period model where some prices are flexible and some are downwards rigid, Citanna, Crès, Drèze, Herings, and Villanacci (2001) find a onedimensional multiplicity of equilibrium that is real.

In a multi-period model of a monetary economy without price stickiness, a model that contains Arrow (1953) as a special case, Drèze and Polemarchakis (2001) find that the dimension of the set of equilibria is equal to the number of terminal date-events, where the multiplicity is entirely nominal. Nakajima and Polemarchakis (2005) extend these ideas to a simple fully articulated two-period macroeconomic model, where the multiplicity is real when producers set prices one period in advance.

From a technical point of view, the main complication in our model is a result of the fact that nominal prices are sticky, which implies that nominal commodity prices have to enter the fixed point argument, and that it is not possible to restrict attention to commodity prices in present-value terms. Since there are no a priori upper bounds on nominal price variables, we consider appropriate limits of economies with compactified price variables. A particular difficulty that has to be addressed is to make sure that the well-known cheaperpoint assumption is satisfied in terms of present-value prices, both for compactified as for limit economies. Cases where exploding nominal commodity prices are offset by Arrow security prices that converge to zero have to be dealt with carefully.

The indeterminacy result can be understood as a simple consequence of counting equations and unknowns. There are as many markets for commodities and assets as there are instruments to clear them, which would suggest that equilibria are determinate. However, there is a budget constraint at the beginning of each date-event and there is a budget constraints at the end of each terminal date-event, where each constraint serves as a Walras' law, and leads to one additional degree of freedom for equilibrium. Since the policy of the bank consists of setting as many interest rates as there are date-events, each interest rate reducing the degrees of freedom by one due to a no-arbitrage condition on asset prices, we are left with the number of terminal date-events as the dimension of the set of equilibria. One channel by which indeterminacy of equilibrium could be reduced is suggested in Magill and Quinzii (2010) and consists of introducing additional instruments for the central bank.

The paper is organized as follows. Section 2 describes the main ingredients of our model - an intertemporal stochastic economy with a general monetary transaction technology and sticky prices - and the concept of sticky price equilibrium. Section 3 explicitly lists all assumptions that are needed for a proof of the general indeterminacy result. Section 4 is devoted to a study of the continuity properties of the budget correspondence. To study 
sticky price equilibria, it is helpful to represent the price and rationing in the market of a single commodity by a single parameter, and to define the concept of a parametrized sticky price equilibrium, which is equivalent to but more tractable than the notion of a sticky price equilibrium. This is the topic of Section 5. In Section 6 we present the main result of the paper about indeterminacy of sticky price equilibria. Section 7 discusses this result and Section 8 considers potential extensions. Section 9 concludes.

\section{The Model}

We provide a formulation of the new neoclassical synthesis that has the same level of generality as the Arrow-Debreu general equilibrium model. There is an event tree $\mathcal{T}$ with the set of date-events $S$ as nodes. The set $S$ is partitioned into subsets $S_{0}, \ldots, S_{T}$, where $S_{t}$ consists of the date-events $s_{t}$ in period $t$. We distinguish between dates and periods, where date $t$ represents the starting point of period $t$ and date $t+1$ its end point. We will also refer to date-events $s_{t}$ and periods $s_{t}$ to distinguish between points and intervals of time. There is a unique date-event $s_{0}$ at $t=0$, the current date-event.

The set of successors of date-event $s_{t}$ is denoted by $s_{t}^{+}$, a subset of $S_{t+1}$. For notational convenience, we introduce a set of date-events $S_{T+1}$ with the same cardinality as $S_{T}$. There is a one-one relationship between date-events in $S_{T}$ and those in $S_{T+1}$. The related dateevent in $S_{T+1}$ corresponds to the end point of period $s_{T}$ and is the unique element of $s_{T}^{+}$. We denote $S^{+}=\cup_{s_{t} \in S} s_{t}^{+}$, so $S^{+}=\left(S \cup S_{T+1}\right) \backslash\left\{s_{0}\right\}$. The unique predecessor of $s_{t} \in S^{+}$ is denoted by $s_{t}^{-}$, an element of $S_{t-1}$.

In each period $s_{t} \in S$ there is trade in a finite set $L$ of commodities by households in a finite set $H$. The price of commodity $\ell$ at date-event $s_{t}$ equals $p_{\ell s_{t}}$. Among other things, the event tree is used to describe when price adjustments take place. Based on a detailed analysis on the distribution of the frequency of price changes in Nakamura and Steinsson (2008), Nakamura and Steinsson (2010) report that the median frequency of monthly price change across sections in the U.S. economy is $8.7 \%$, implying that the median duration of a particular price across sectors is around one year. These authors also report considerable heterogeneity in this frequency across sectors. For most commodities, therefore, price adjustments do not take place at every period. As in Debreu (1959), the event tree is sufficiently refined for all prices to be constant in period $s_{t}$.

At each date-event $s_{t} \in S$, the price of commodity $\ell \in L$ is either sticky or can be adjusted. For each $\ell \in L$, this leads to a partition of $S$ consisting of the sets $N_{\ell}^{\mathrm{s}}$ and $N_{\ell}^{\mathrm{a}}$. The price of commodity $\ell$ can be adjusted at a date-event $s_{t} \in N_{\ell}^{\mathrm{a}}$. The set $N_{\ell}^{\mathrm{s}}$ consists of those date-events, where the price of commodity $\ell$ is sticky, and therefore equal to $p_{\ell s_{t}^{-}}$. When the price of commodity $\ell$ is sticky at date-event $s_{0}$, it is inherited from the price 
$p_{\ell s_{-1}}$ set at date-event $s_{-1}$, a price that is exogenously given at $s_{0}$. We allow for the case where the price of commodity $\ell$ is flexible at all date-events, in which case $N_{\ell}^{\mathrm{a}}=S$, the case where the price of commodity $\ell$ is fixed at all date-events, $N_{\ell}^{\mathrm{a}}=\emptyset$, as well as all the intermediate cases. The specification in Taylor (1980), where prices are sticky for a fixed number of periods, and the specification of Calvo (1983), where it is determined by chance whether a price can be adjusted, are both obtained as special cases.

Since the price of commodity $\ell$ at date-events in $N_{\ell}^{\mathrm{s}}$ is sticky, the markets of these commodities are cleared by quantity adjustments. Violations of voluntary trading are not allowed for. This deviates from part of the macroeconomic literature where quantity adjustments are made by forcing the short side of the market to accommodate the trades desired by the long side. Such an approach is not feasible in our general set-up. In our model, trading on a particular commodity market is not only influenced by the price, but also by the maximal amount a household is able to supply of every commodity, called the rationing scheme on supply, and by the maximal amount a household is able to demand for every commodity, called the rationing scheme on demand. These constraints are imposed on the long side of the market and are determined by the short side. Rationing schemes serve as the matching technology between supply and demand. Since markets are assumed to be fully transparent, rationing affects the long side of the market only.

Rationing can take many forms. For the sake of simplicity, we consider uniform rationing, implying that the rationing scheme on supply is described by $\underline{z} \in-\mathbb{R}_{+}^{* L S}$ and the rationing scheme on demand by $\bar{z} \in \mathbb{R}_{+}^{* L S}$, where $\mathbb{R}^{*}=\mathbb{R} \cup\{+\infty\}$ denotes the set of extended real numbers. We model the absence of constraints on a particular market $\ell s_{t}$ by setting $\underline{z}_{\ell_{t}}=-\infty$ and $\bar{z}_{\ell_{t}}=+\infty$, for instance at date-events in $N_{\ell}^{\text {a }}$, where rationing does not take place at equilibrium. The way rationing is modeled is taken from the approach used by Drèze (1975) to study general equilibrium models with price rigidities, see Herings (1996b) for a general treatment. In the macroeconomic literature, such an approach is taken for instance in Svensson (1986). The values of the variables $\underline{z}$ and $\bar{z}$ are determined endogenously in an equilibrium.

Price stickiness involves nominal prices. For nominal prices to be meaningful, we need to extend the model by a monetary sector. To a large extent, we follow the monetary sector model of Drèze and Polemarchakis (2001), a model that is compatible with Chapter 2 of Woodford (2003), and that can be viewed as its general equilibrium extension. Households hold money for transaction purposes and have a bank loan that is adjusted whenever withdrawels or deposits of money are made.

On top of the real and the monetary part, we assume sequentially complete asset markets, where households trade Arrow securities. Contrary to what is common in the macroeconomic literature, we will not resort to loglinearizations, but rather consider the 
actual supply and demand correspondences of commodities, money, and assets.

The timing of our model is as follows. A period $s_{t} \in S$ starts at date $t$ with transactions on the asset markets. Asset market transactions involve buying and selling Arrow securities contingent on future date-events and collecting dividends from the asset portfolio held in the previous period. Households use the proceeds from asset market transactions to adjust holdings of the bank loan and money balances. At dates in the interval $(t, t+1)$, transactions on the spot markets for commodities take place against money, and transactions with the bank occur exchanging bank loans against money. At date $t+1$, period $s_{t}$ terminates with the payment of interest due to the bank and the collection of the bank seignorage by the bank's shareholders.

A more detailed account of the monetary part of the model is as follows. At each period $s_{t} \in S$, the bank offers loan and deposit facilities to households against a non-negative interest rate $r_{s_{t}}$. The bank supplies money balances as demanded by the households. At $\tau \in[t, t+1]$, household $h$ has a bank loan $b_{s_{t}}^{h}(\tau)$ and holds money balances $m_{s_{t}}^{h}(\tau)$. A withdrawal of money balances from the bank by the household increases the bank loan by the same amount, whereas a deposit of money at the bank leads to a decrease of the bank loan by the same magnitude. Transactions with other households increase money balances by an amount equal to the value of net sales, but do not affect the bank loan. At the end of period $s_{t}$, the average bank loan of household $h$ in period $s_{t}, b_{s_{t}}^{h}=\int_{\tau=t}^{t+1} b_{s_{t}}^{h}(\tau) d \tau$, gives rise to interest payments equal to $r_{s_{t}} b_{s_{t}}^{h}$. We do allow for the special case where all interest rates are equal to zero. When interest rates are all zero and all prices can be adjusted at all date-events, the real part of our model reduces to Arrow (1953).

A more detailed account of the asset market part of the model follows next. At each date-event $s_{t} \in S$ there are $\left|s_{t}^{+}\right|$Arrow securities, one for each date-event in $s_{t}^{+}$. An Arrow security for date-event $s_{t+1}$ is traded at date-event $s_{t+1}^{-}$against a price $q_{s_{t+1}}$ and pays one nominal unit if and only if date-event $s_{t+1}$ occurs. Because of the availability of Arrow securities, markets are sequentially complete. A standard no-arbitrage argument implies that at equilibrium the sum of the prices of the Arrow securities traded at date-event $s_{t}$ must be equal to $1 /\left(1+r_{s_{t}}\right)$. At no-arbitrage prices, asset demand is indeterminate as any household is indifferent between holding one unit less of the bank loan and one unit more of every Arrow security. To lift this indeterminacy, we set beginning-of-period bank loans equal to money balances for every household, $b_{s_{t}}^{h}(t)=m_{s_{t}}^{h}(t)$. Notice that we distinguish between end-of-period bank loans $b_{s_{t}^{-}}^{h}(t)$ at $s_{t}^{-}$, where a discrepancy between $b_{s_{t}^{-}}^{h}(t)$ and $m_{s_{t}^{-}}^{h}(t)$ is possible, and beginning-of-period bank loans $b_{s_{t}}^{h}(t)$ at $s_{t}$, which are equal to $m_{s_{t}}^{h_{t}}(t)$ by definition.

Aggregate money balances issued by the bank at $\tau$ are $m_{s_{t}}^{\mathrm{c}}(\tau)=\sum_{h \in H} m_{s_{t}}^{h}(\tau)$, a nonnegative quantity. Average aggregate money balances issued in period $s_{t}$ equal $m_{s_{t}}^{\mathrm{c}}=$ 
$\int_{\tau=t}^{t+1} m_{s_{t}}^{\mathrm{c}}(\tau) d \tau$. Since beginning-of-period bank loans are set equal to money balances for every household, it holds that $b_{s_{t}}^{\mathrm{c}}(\tau)=\sum_{h \in H} b_{s_{t}}^{h}(\tau)=\sum_{h \in H} m_{s_{t}}^{h}(\tau)$ and $b_{s_{t}}^{\mathrm{c}}=\sum_{h \in H} b_{s_{t}}^{h}=$ $\sum_{h \in H} m_{s_{t}}^{h}$. At the end of period $s_{t}$, the bank collects an amount $v_{s_{t}}^{\mathrm{c}}=r_{s_{t}} b_{s_{t}}^{\mathrm{c}}$ of interest payments as seignorage. The bank issues the entire seignorage as dividends to its shareholders at the end of the period. Household $h$ receives $v_{s_{t}}^{h}=\theta^{h} v_{s_{t}}^{\mathrm{c}}$ at the end of period $s_{t}$ with $\theta^{h}$ the shareholdings of household $h$.

At date-event $s_{t} \in S$, i.e. at the beginning of period $s_{t}$, household $h$ has wealth given by the returns from investments in Arrow securities in the previous period $a_{s_{t}}^{h}$, plus monetary holdings at the end of the previous period $m_{s_{t}^{-}}^{h}(t)$, minus the bank loan at the end of the previous period $b_{s_{t}^{-}}^{h}(t)$. Making use of the property that $b_{s_{t}^{-}}^{h}(t-1)=m_{s_{t}^{-}}^{h}(t-1)$, it follows that the monetary holdings minus the bank loan at the end of the previous period equal net sales of commodities in the previous period plus dividends received minus interest payments, $m_{s_{t}^{-}}^{h}(t)-b_{s_{t}^{-}}^{h}(t)=p_{s_{t}^{-}}\left(e_{s_{t}^{-}}^{h}-x_{s_{t}^{-}}^{h}\right)+v_{s_{t}^{-}}^{h}-r_{s_{t}^{-}} b_{s_{t}^{-}}^{h}$.

Household $h$ invests his wealth at date-event $s_{t}$ in Arrow securities $a_{s_{t+1}}^{h}$, where $s_{t+1} \in$ $s_{t}^{+}$. It follows that household $h$ faces the following sequence of budget constraints,

$$
\begin{array}{rlrl}
\sum_{s_{1} \in s_{0}^{+}} q_{s_{1}} a_{s_{1}}^{h}+m_{s_{0}}^{h}(0)-b_{s_{0}}^{h}(0) & =0, & \\
\sum_{s_{t+1} \in s_{t}^{+}} q_{s_{t+1}} a_{s_{t+1}}^{h}+m_{s_{t}}^{h}(t)-b_{s_{t}}^{h}(t) & =a_{s_{t}}^{h}+m_{s_{t}^{-}}^{h}(t)-b_{s_{t}^{-}}^{h}(t), & & s_{t} \in S \backslash\left\{s_{0}\right\}, \\
a_{s_{T+1}}^{h}+m_{s_{T+1}^{-}}^{h}(T+1)-b_{s_{T+1}^{-}}^{h}(T+1) & =0, & & s_{T+1} \in S_{T+1},
\end{array}
$$

the lifting-of-indeterminacy identities

$$
b_{s t}^{h}(t)=m_{s t}^{h}(t), \quad s_{t} \in S
$$

and the accounting identities

$$
m_{s_{t}}^{h}(t+1)-b_{s_{t}}^{h}(t+1)=p_{s_{t}}\left(e_{s_{t}}^{h}-x_{s_{t}}^{h}\right)+v_{s_{t}}^{h}-r_{s_{t}} b_{s_{t}}^{h}, \quad s_{t} \in S
$$

Substitution of the accounting identities and the lifting-of-indeterminacy identities in the budget constraints eliminates the monetary balances $m_{s_{t}}^{h}(t)$ and bank loan holdings $b_{s_{t}}^{h}(t)$. The only relevant aspects of the monetary transactions are the interest payments $r_{s_{t}} b_{s_{t}}^{h}$ made by household $h$ at the end of period $s_{t}$.

The value of $b^{h}$ is determined by the transaction technology correspondence $\beta^{h}: \mathbb{R}_{+}^{L S} \times$ $X^{h} \rightarrow \mathbb{R}^{S}$ of household $h$. It assigns to each $p \in \mathbb{R}_{+}^{L S}$ and consumption bundle $x^{h} \in X^{h}$ a subset $\beta^{h}\left(p, x^{h}\right)$ of $\mathbb{R}^{S}$. An element $b^{h}$ of $\beta^{h}\left(p, x^{h}\right)$ specifies the bank loans that are sufficient to carry out purchases and sales involved in consumption of $x^{h}$ when prices are $p$. A typical example concerns a Clower (1967) type cash-in-advance technology, where $b^{h} \in \beta^{h}\left(p, x^{h}\right)$ if and only if $b_{s_{t}}^{h} \geq p_{s_{t}} x_{s_{t}}^{h}, s_{t} \in S$. Alternatively, when cash is only needed for net purchases, $b^{h} \in \beta^{h}\left(p, x^{h}\right)$ if and only if $b_{s_{t}}^{h} \geq p_{s_{t}}\left(x_{s_{t}}^{h}-e_{s_{t}}^{h}\right)^{+}, s_{t} \in S$, where for a real-valued vector 
$z$ we use the notation $z^{+}=\max \{0, z\} .{ }^{1}$ Both specifications make the implicit assumption that cash needed for purchases is needed in advance, whereas cash resulting from sales is only available at the end of the period. If, instead, cash resulting from sales is immediately available for purchases, then the natural specification becomes $b^{h} \in \beta^{h}\left(p, x^{h}\right)$ if and only if $b_{s_{t}}^{h} \geq\left(p_{s_{t}}\left(x_{s_{t}}^{h}-e_{s_{t}}^{h}\right)\right)^{+}, s_{t} \in S$.

As in Lucas and Stokey (1987), we can incorporate the distinction between "cash goods" which are subject to a cash-in-advance constraint, and "credit goods" which do not need to be paid for in cash as would be the case for instance for leisure, and it is entirely possible to make the cash requirements good specific. The transaction technology can be made state-dependent, which enables us to model that the monetary transactions technology is subject to velocity shocks.

The modeling of the transaction technology incorporates a rich variety of other specifications, and allows for specifications where households have interest elastic money demand as in the Baumol-Tobin model developed independently in Baumol (1952) and Tobin (1956). All that is needed is to have one of the commodities $\ell$ representing cash-withdrawal services, the consumption of which diminishes the need for cash balances.

The transaction technology correspondence approach avoids specifications where prices enter the utility function. Utility is derived from the consumption of goods, and the only way money holdings and prices affect utilities is via the commodities that can be purchased.

The description of an economy $\mathcal{E}=\left(\mathcal{T},\left(X^{h}, \preceq^{h}, e^{h}, \theta^{h}, \beta^{h}\right)_{h \in H},\left(N_{\ell}^{\mathrm{a}}, N_{\ell}^{\mathrm{s}}\right)_{\ell \in L}, p_{s_{-1}}, r\right)$ is completed by a specification of $\left(\preceq_{h}\right)_{h \in H}$, with $\preceq^{h}$ the preference relation of household $h$ defined on $X^{h}$, and a specification of prices $p_{s_{-1}}$ at date-event $s_{-1}$, where only the prices $p_{\ell s_{-1}}$ for $\ell$ such that $s_{0} \in N_{\ell}^{\mathrm{s}}$ matter.

The monetary part of the model deviates from the treatment in Drèze and Polemarchakis (2001) in two, relatively minor, aspects. The transaction technology there is a correspondence that assigns a set of feasible consumption bundles and bank loans $\left(x^{h}, b^{h}\right)$ to each price system $p$. In this paper, we assign a set of feasible bank loans $b^{h}$ to each price system and consumption bundle $\left(p, x^{h}\right)$. A second, more substantial, difference is that we allow bank loans to be negative, which would naturally occur when a household makes many sales in a particular period, resulting in a bank deposit rather than a bank loan.

A household takes prices $(p, q)$, interest rates $r$, rationing schemes $(-\underline{z}, \bar{z})$, and dividends $v^{h}$ as given, and chooses a maximal element $\left(x^{h}, a^{h}, b^{h}\right)$ for $\preceq^{h}$ subject to the constraints

\footnotetext{
${ }^{1}$ The maximum of two vectors is defined by taking the componentwise maximum. Similarly, the minimum of two vectors is defined by taking the componentwise minimum.
} 
$x^{h} \in X^{h}, b^{h} \in \beta^{h}\left(p, x^{h}\right), \underline{z} \leq x^{h}-e^{h} \leq \bar{z}$, and the sequence of budget constraints

$$
\begin{array}{rlrl}
\sum_{s_{1} \in s_{0}^{+}} q_{s_{1}} a_{s_{1}}^{h} & =0 & & \\
\sum_{s_{t+1} \in s_{t}^{+}} q_{s_{t+1}} a_{s_{t+1}}^{h} & =a_{s_{t}}^{h}-p_{s_{t}^{-}}\left(x_{s_{t}^{-}}^{h}-e_{s_{t}^{-}}^{h}\right)-r_{s_{t}^{-}} b_{s_{t}^{-}}^{h}+v_{s_{t}^{-}}^{h}, & & s_{t} \in S \backslash\left\{s_{0}\right\}, \\
0 & =a_{s_{T+1}}^{h}-p_{s_{T+1}^{-}}\left(x_{s_{T+1}^{-}}^{h}-e_{s_{T+1}^{-}}^{h}\right)-r_{s_{T+1}^{-}} b_{s_{T+1}^{-}}^{h}+v_{s_{T+1}^{-}}^{h}, & s_{T+1} \in S_{T+1} .
\end{array}
$$

The budget set $\gamma^{h}\left(p, q, r, \underline{z}, \bar{z}, v^{h}\right)$ consists of all tuples $\left(x^{h}, a^{h}, b^{h}\right)$ satisfying these constraints.

We use the notational convention that $x$ is indexed by $h \in H, \ell \in L$, and $s_{t} \in S, b$ and $v$ are indexed by $h \in H$ and $s_{t} \in S, a$ is indexed by $h \in H$ and $s_{t} \in S^{+}, p, \underline{z}$ and $\bar{z}$ by $\ell \in L$ and $s_{t} \in S$, and $q$ by $s_{t} \in S^{+}$.

Definition 2.1 A sticky price equilibrium for the economy $\mathcal{E}$ is $\left(p^{*}, q^{*}, \underline{z}^{*}, \bar{z}^{*}, v^{*}, x^{*}, a^{*}, b^{*}\right)$ in $\mathbb{R}^{L S} \times \mathbb{R}^{S^{+}} \times-\mathbb{R}_{+}^{* L S} \times \mathbb{R}_{+}^{* L S} \times \mathbb{R}^{H S} \times \mathbb{R}^{H L S} \times \mathbb{R}^{H S^{+}} \times \mathbb{R}^{H S}$ such that

(a) for $h \in H,\left(x^{* h}, a^{* h}, b^{* h}\right)$ is $\preceq^{h}$-maximal on $\gamma^{h}\left(p^{*}, q^{*}, r, \underline{z}^{*}, \bar{z}^{*}, v^{* h}\right)$,

(b) commodity markets clear, $\sum_{h \in H} x^{* h}=\sum_{h \in H} e^{h}$,

(c) Arrow security markets clear, $\sum_{h \in H} a^{* h}=0$,

(d) the no-arbitrage conditions hold, for $s_{t} \in S, \sum_{s_{t+1} \in s_{t}^{+}} q_{s_{t+1}}^{*}=1 /\left(1+r_{s_{t}}\right)$,

(e) for $h \in H, s_{t} \in S$, dividends satisfy $v_{s_{t}}^{* h}=\theta^{h} r_{s_{t}} \sum_{h \in H} b_{s_{t}}^{* h}$,

(f) for $s_{t} \in N_{\ell}^{\mathrm{s}}$, prices equal the previous period's value, $p_{\ell s_{t}}^{*}=p_{\ell s_{t}^{-}}^{*}$,

(g) no rationing when the price is flexible, for $\ell \in L, s_{t} \in N_{\ell}^{\mathrm{a}}, \underline{z}_{\ell s_{t}}^{*}=-\infty$ and $\bar{z}_{\ell s_{t}}^{*}=+\infty$,

(h) rationing is one-sided, for $\ell \in L, s_{t} \in N_{\ell}^{\mathrm{s}}$,

$$
\begin{array}{lll}
\underline{z}_{\ell s_{t}}^{*}>-\infty & \text { implies } & \bar{z}_{\ell s_{t}}^{*}=+\infty \\
\bar{z}_{\ell s_{t}}^{*}<+\infty & \text { implies } \quad \underline{z}_{\ell s_{t}}^{*}=-\infty .
\end{array}
$$

In Definition 2.1 there is no reference to the variables $b_{s_{t}}^{\mathrm{c}}$ and $m_{s_{t}}^{\mathrm{c}}$. They follow from the accounting identities $b_{s_{t}}^{\mathrm{c}}=\sum_{h \in H} b_{s_{t}}^{h}$ and $m_{s_{t}}^{\mathrm{c}}=b_{s_{t}}^{\mathrm{c}}$.

A household is not necessarily influenced by the constraints on his choices caused by the rationing scheme $(\underline{z}, \bar{z})$. A household $h$ is constrained on his supply in the market for contingent commodity $\ell s_{t}$ at $\left(p, q, r, \underline{z}, \bar{z}, v^{h}\right)$ if there exists $\left(\hat{x}^{h}, \hat{a}^{h}, \hat{b}^{h}\right) \in \gamma^{h}\left(p, q, r, \underline{\hat{z}}, \bar{z}, v^{h}\right)$, where $\underline{\hat{z}}$ equals $\underline{z}$, except that $\underline{\hat{z}}_{l s_{t}}=-\infty$, such that for all $\left(x^{h}, a^{h}, b^{h}\right) \in \gamma^{h}\left(p, q, r, \underline{z}, \bar{z}, v^{h}\right), \hat{x}^{h}$ is strictly preferred to $x^{h}$. The definition for a household to be constrained on his demand in 
the market for contingent commodity $\ell s_{t}$ is analogous. There is supply (demand) rationing in the market for contingent commodity $\ell s_{t}$ at $\left(p, q, r, \underline{z}, \bar{z}, v^{h}\right)$ if at least one household is constrained on his supply (demand) in the market for commodity $\ell s_{t}$ at $\left(p, q, r, \underline{z}, \bar{z}, v^{h}\right)$. There is rationing in the market for contingent commodity $\ell s_{t}$ at $\left(p, q, r, \underline{z}, \bar{z}, v^{h}\right)$ if there is supply rationing or demand rationing in the market for commodity $\ell s_{t}$ at $\left(p, q, r, \underline{z}, \bar{z}, v^{h}\right)$.

For the sake of simplicity, we have presented a model of a pure exchange economy. It is a routine exercise to extend the model and the sticky price equilibrium concept to a production economy.

\section{Assumptions}

The assumptions below are made throughout the paper without further mentioning.

A1. For $h \in H, X^{h}$ is closed, convex, has a lower bound, and $X^{h}+\mathbb{R}_{+}^{L S} \subset X^{h}$. There exists $x^{h} \in X^{h}$ such that $x^{h} \ll e^{h}$.

A2. For $h \in H, \preceq^{h}$ is transitive, complete, continuous, convex, and monotonic: if $x^{h}, \bar{x}^{h} \in$ $X^{h}$ with $x^{h}<\bar{x}^{h}$, then $x^{h} \prec^{h} \bar{x}^{h}$.

A3. The bank is owned by the households: for $h \in H, \theta^{h} \geq 0$, and $\sum_{h \in H} \theta^{h}=1$.

A4. For $h \in H$ we have:

1. The correspondence $\beta^{h}$ is lower hemi-continuous and closed.

2. Monetary needs are bounded: there exist continuous functions $\underline{f}^{h}: \mathbb{R}_{+}^{L S} \times X^{h} \rightarrow$ $-\mathbb{R}_{+}^{S}, \bar{f}^{h}: \mathbb{R}_{+}^{L S} \times X^{h} \rightarrow \mathbb{R}_{+}^{S}$ such that $b^{h} \in \beta^{h}\left(p, x^{h}\right)$ implies $\underline{f}^{\bar{h}}\left(p, x^{h}\right) \leq b^{h}$ and $\min \left\{\bar{f}^{h}\left(p, x^{h}\right), b^{h}\right\} \in \beta^{h}\left(p, x^{h}\right)$.

3. The correspondence $\beta^{h}$ is homogeneous in prices. Consider $p, \bar{p} \in \mathbb{R}_{+}^{L S}$ and $\lambda \geq 0$ such that $\bar{p}_{\bar{s}_{t}}=\lambda p_{\bar{s}_{t}}$ and, for $s_{t} \in S \backslash\left\{\bar{s}_{t}\right\}, \bar{p}_{s_{t}}=p_{s_{t}}$. Consider $x^{h} \in X^{h}$. Then it holds that $b^{h} \in \beta^{h}\left(p, x^{h}\right)$ implies $\bar{b}^{h} \in \beta^{h}\left(\bar{p}, x^{h}\right)$, where $\bar{b}_{\bar{s}_{t}}^{h}=\lambda b_{\bar{s}_{t}}^{h}$ and, for $s_{t} \in S \backslash\left\{\bar{s}_{t}\right\}, \bar{b}_{s_{t}}^{h}=b_{s_{t}}^{h}$.

4. The correspondence $\beta^{h}$ satisfies the following convexity property: for $p \in \mathbb{R}_{+}^{L S}$, for $x^{h}, \bar{x}^{h} \in X^{h}$, it holds that $b^{h} \in \beta^{h}\left(p, x^{h}\right)$ and $\bar{b}^{h} \in \beta^{h}\left(p, \bar{x}^{h}\right)$ implies $\lambda b^{h}+$ $(1-\lambda) \bar{b}^{h} \in \beta^{h}\left(p, \lambda x^{h}+(1-\lambda) \bar{x}^{h}\right)$ for all $\lambda \in[0,1]$.

5. For $p \in \mathbb{R}_{+}^{L S}$, for $x^{h} \in X^{h}, x^{h} \leq e^{h}$ implies $0 \in \beta^{h}\left(p, x^{h}\right)$.

6. For $p \in \mathbb{R}_{+}^{L S}$, for $\ell s_{t} \in L \times S$ with $p_{\ell s_{t}}=0$, for $x^{h}, \bar{x}^{h} \in X^{h}$ with $\bar{x}^{h}=x^{h}+\varepsilon e_{\ell s_{t}}$ for some $\varepsilon>0$, it holds that $\beta^{h}\left(p, x^{h}\right) \subset \beta^{h}\left(p, \bar{x}^{h}\right)$. 
A5. Only the bank can create money: if $x \in \prod_{h} X^{h}$ satisfies $\sum_{h} x^{h}=\sum_{h} e^{h}$ and, for some $p \in \mathbb{R}_{+}^{L S}$, for all $h \in H, b^{h} \in \beta^{h}\left(p, x^{h}\right)$, then $\sum_{h \in H} b^{h} \geq 0$.

A6. $p_{s_{-1}} \gg 0$.

The Assumptions A1, A2, A3, and A6 are standard. A4.1 is a standard continuity assumption that is satisfied by cash-in-advance technologies for instance. We require $\beta^{h}$ to be closed rather than to be upper hemi-continuous, since the latter assumption is quite strong for correspondences that are not compact-valued, and would be violated by cash-inadvance technologies. Assumption A4.2 puts lower and upper bounds on monetary needs. A natural choice for the functions $\underline{f}^{h}, \bar{f}^{h}: \mathbb{R}_{+}^{L S} \times X^{h} \rightarrow \mathbb{R}_{+}^{S}$ would be

$$
\begin{aligned}
f_{s_{t}}^{h}\left(p, x^{h}\right)=-p_{s_{t}}\left(e_{s_{t}}^{h}-x_{s_{t}}^{h}\right)^{+}, & s_{t} \in S, \\
\bar{f}_{s_{t}}^{h}\left(p, x^{h}\right) & =p_{s_{t}}\left(x_{s_{t}}^{h}-e_{s_{t}}^{h}\right)^{+}, \quad s_{t} \in S .
\end{aligned}
$$

Assumption A4.3 is a standard homogeneity assumption, and A4.4 a standard convexity assumption. In A4.5 we require that when a consumption bundle involves only sales, no bank loan is needed. This is natural, since making the sales should result in a bank deposit rather than a loan. Similarly, for a consumption bundle involving only purchases, non-negative bank loans are required. A4.6 states that additional consumption of a commodity with a zero price does not require additional money balances. ${ }^{2}$ A5 requires that all attainable allocations involve non-negative aggregate monetary holdings.

\section{Continuity of Budget Correspondences}

How does one prove the existence of a sticky price equilibrium as defined in Definition 2.1? The first problem to be taken care of is a continuity problem. The budget correspondence may fail to be continuous at $\left(p, q, r, \underline{z}, \bar{z}, v^{h}\right)$. To facilitate the study of continuity, we rewrite the sequence of budget constraints into a single budget constraint in terms of present-value prices.

We denote the present-value price at $s_{0}$ of one unit of income at date-event $s_{t} \in\left\{s_{0}\right\} \cup S^{+}$ by $q_{s_{t}}^{0}$. With $s_{t}\left(s_{t^{\prime}}\right)$ denoting the predecessor of $s_{t^{\prime}}$ at date $t$, we have

$$
\begin{aligned}
& q_{s_{0}}^{0}=1, \\
& q_{s_{t}}^{0}=q_{s_{1}\left(s_{t}\right)} q_{s_{2}\left(s_{t}\right)} \cdots q_{s_{t-1}\left(s_{t}\right)} q_{s_{t}}, \quad s_{t} \in S^{+} .
\end{aligned}
$$

The sequence of budget constraints can be rewritten in a more convenient way. When we multiply the budget constraint at date-event $s_{t}$ by $q_{s_{t}}^{0}$ and add up we get

$$
\sum_{s_{t} \in S} q_{s_{t}}^{0} \sum_{s_{t+1} \in s_{t}^{+}} q_{s_{t+1}} a_{s_{t+1}}^{h}=\sum_{s_{t} \in S^{+}} q_{s_{t}}^{0}\left(a_{s_{t}}^{h}-p_{s_{t}^{-}}\left(x_{s_{t}^{-}}^{h}-e_{s_{t}^{-}}^{h}\right)-r_{s_{t}^{-}} b_{s_{t}^{-}}^{h}+v_{s_{t}^{-}}^{h}\right) .
$$

\footnotetext{
${ }^{2}$ If this commodity helps in saving on transaction costs, more consumption could actually decrease the amount of money balances needed, a possibility that is allowed for in the current formulation.
} 
After canceling the $a^{h}$-terms which appear on both sides with identical multiplicands, and rearranging terms, we obtain

$$
\sum_{s_{t} \in S^{+}} q_{s_{t}}^{0}\left(p_{s_{t}^{-}} x_{s_{t}^{-}}^{h}+r_{s_{t}^{-}} b_{s_{t}^{-}}^{h}\right)=\sum_{s_{t} \in S^{+}} q_{s_{t}}^{0}\left(p_{s_{t}^{-}} e_{s_{t}^{-}}^{h}+v_{s_{t}^{-}}^{h}\right) .
$$

Since $\sum_{s_{t+1} \in s_{t}^{+}} q_{s_{t+1}}=1 /\left(1+r_{s_{t}}\right)$, we find

$$
\sum_{s_{t} \in S}\left(\frac{q_{s_{t}}^{0}}{1+r_{s_{t}}} p_{s_{t}} x_{s_{t}}^{h}+\frac{q_{s_{t}}^{0}}{1+r_{s_{t}}} r_{s_{t}} b_{s_{t}}^{h}\right)=\sum_{s_{t} \in S}\left(\frac{q_{s_{t}}^{0}}{1+r_{s_{t}}} p_{s_{t}} e_{s_{t}}^{h}+\frac{q_{s_{t}}^{0}}{1+r_{s_{t}}} v_{s_{t}}^{h}\right) .
$$

It is now straightforward to verify that the original sequence of budget constraints is equivalent to (4.1) plus the recursive system of equations

$$
\begin{array}{rlrl}
a_{s_{T+1}}^{h} & =p_{s_{T+1}^{-}}\left(x_{s_{T+1}^{-}}^{h}-e_{s_{T+1}^{h}}^{h}\right)+r_{s_{T+1}^{-}} b_{s_{T+1}^{-}}^{h}-v_{s_{T+1}^{-}}^{h}, & & s_{T+1} \in S_{T+1}, \\
a_{s_{t}}^{h} & =p_{s_{t}^{-}}\left(x_{s_{t}^{-}}^{h}-e_{s_{t}^{-}}^{h}\right)+r_{s_{t}^{-}} b_{s_{t}^{-}}^{h}-v_{s_{t}^{-}}^{h}+\sum_{s_{t+1} \in s_{t}^{+}} q_{s_{t+1}} a_{s_{t+1}}^{h}, & s_{t} \in S \backslash\left\{s_{0}\right\} .
\end{array}
$$

Since the $a^{h}$-terms are neither part of the preferences nor of any of the other constraints, the consumer choice problem has been reduced to the choice of $\left(x^{h}, b^{h}\right)$ with $x^{h} \in X^{h}$ and $b^{h} \in \beta^{h}\left(p, x^{h}\right)$ subject to the single budget constraint (4.1) and the quantity constraints $\underline{z} \leq x^{h}-e^{h} \leq \bar{z}$. Moreover, by substituting $\tilde{q}_{s t}=q_{s_{t}}^{0} /\left(1+r_{s_{t}}\right), \tilde{p}_{s_{t}}=\tilde{q}_{s_{t}} p_{s_{t}}, \tilde{b}_{s_{t}}^{h}=\tilde{q}_{s_{t}} b_{s_{t}}^{h}$, and $\tilde{v}_{s_{t}}^{h}=\tilde{q}_{s_{t}} v_{s_{t}}^{h}$, the budget constraint (4.1) can be rewritten as

$$
\sum_{s_{t} \in S}\left(\tilde{p}_{s_{t}} x_{s_{t}}^{h}+r_{s_{t}} \tilde{b}_{s_{t}}^{h}\right)=\sum_{s_{t} \in S}\left(\tilde{p}_{s_{t}} e_{s_{t}}^{h}+\tilde{v}_{s_{t}}^{h}\right)
$$

or, even shorter,

$$
\tilde{p} x^{h}+r \tilde{b}^{h}=\tilde{p} e^{h}+\tilde{w}^{h}
$$

where $\tilde{w}^{h}=\sum_{s_{t} \in S} \tilde{v}_{s t}^{h}$.

We refer to prices $\tilde{p}$ as present-value prices, and, more generally, refer to variables with a tilde as present-value variables. Although our definition for $\tilde{p}$ is the most convenient one, it deviates from what is also referred to as a present-value price, the variable $p^{0}$ defined by $p_{s_{t}}^{0}=q_{s_{t}}^{0} p_{s_{t}}$ for $s_{t} \in S$. To see that $p^{0}$ is also an appropriate present-value price in our model, consider the case of a cash-in-advance constraint with $b_{s_{t}}^{h}=p_{s_{t}} x_{s_{t}}^{h}$. We see that the budget constraint (4.1) reduces to

$$
\sum_{s_{t} \in S} p_{s_{t}}^{0} x_{s_{t}}^{h}=\sum_{s_{t} \in S}\left(\frac{1}{1+r_{s_{t}}} p_{s_{t}}^{0} e_{s_{t}}^{h}+\tilde{v}_{s_{t}}^{h}\right)
$$

The right-hand side of this constraint consists entirely of variables which are exogenous to the household. The prices relevant for the purchase of consumption goods are given by $p^{0}$. 
When we consider the cash-in-advance constraint on net trades, $b_{s_{t}}^{h}=p_{s_{t}}\left(x_{s_{t}}^{h}-e_{s_{t}}^{h}\right)^{+}$, we can rewrite the budget constraint (4.1) to

$$
\sum_{s_{t} \in S} p_{s_{t}}^{0}\left(x_{s_{t}}^{h}-e_{s_{t}}^{h}\right)^{+}=\sum_{s_{t} \in S}\left(\frac{1}{1+r_{s_{t}}} p_{s_{t}}^{0}\left(e_{s_{t}}^{h}-x_{s_{t}}^{h}\right)^{+}+\tilde{v}_{s_{t}}^{h}\right) .
$$

In this case we obtain a wedge between buying prices $p_{s_{t}}^{0}$ and selling prices $\left(1 /\left(1+r_{s_{t}}\right)\right) p_{s_{t}}^{0}$. Positive nominal interest rates create distortions and cause equilibrium marginal rates of substitution of households to differ from one another, thereby leading to absence of Pareto optimality even in the absence of price stickiness.

It is convenient to introduce the set $\bar{Q}$ of prices of Arrow securities that are arbitragefree,

$$
\bar{Q}=\left\{(q, r) \in \mathbb{R}_{+}^{S^{+}} \times \mathbb{R}_{+}^{S} \mid \forall s_{t} \in S, \quad \sum_{s_{t+1} \in s_{t}^{+}} q_{s_{t+1}}=\frac{1}{1+r_{s_{t}}}\right\} .
$$

We define the correspondence $\hat{\gamma}^{h}: \mathbb{R}_{+}^{L S} \times \bar{Q} \times-\mathbb{R}_{+}^{L S} \times \mathbb{R}_{+}^{L S} \times \mathbb{R}_{+}^{S} \rightarrow X^{h} \times \mathbb{R}^{S^{+}} \times \mathbb{R}^{S}$ by

$$
\begin{aligned}
& \hat{\gamma}^{h}\left(p, q, r, \underline{z}, \bar{z}, v^{h}\right)=\left\{\left(x^{h}, a^{h}, b^{h}\right) \in X^{h} \times \mathbb{R}^{S^{+}} \times \mathbb{R}^{S} \mid\right. \\
& b^{h} \in \beta^{h}\left(p, x^{h}\right) \text {, } \\
& \underline{z} \leq x^{h}-e^{h} \leq \bar{z} \text {, } \\
& \tilde{p} x^{h}+r \tilde{b}^{h} \leq \tilde{p} e^{h}+\tilde{w}^{h}, \\
& a_{s_{T+1}}^{h}=p_{s_{T+1}^{-}}\left(x_{s_{T+1}^{-}}^{h}-e_{s_{T+1}^{-}}^{h}\right)+r_{s_{T+1}^{-}} b_{s_{T+1}^{-}}^{h}-v_{s_{T+1}^{-}}^{h}, \quad s_{T+1} \in S_{T+1} \text {, } \\
& \left.a_{s_{t}}^{h}=p_{s_{t}^{-}}\left(x_{s_{t}^{-}}^{h}-e_{s_{t}^{-}}^{h}\right)+r_{s_{t}^{-}} b_{s_{t}^{-}}^{h}-v_{s_{t}^{-}}^{h}+\sum_{s_{t+1} \in s_{t}^{+}} q_{s_{t+1}} a_{s_{t+1}}^{h}, \quad s_{t} \in S \backslash\left\{s_{0}\right\}\right\} \text {, }
\end{aligned}
$$

and the correspondence $\tilde{\gamma}^{h}: \mathbb{R}_{+}^{L S} \times \mathbb{R}_{+}^{S} \times-\mathbb{R}_{+}^{L S} \times \mathbb{R}_{+}^{L S} \times \mathbb{R}_{+} \rightarrow X^{h} \times \mathbb{R}^{S}$ by

$$
\begin{aligned}
\tilde{\gamma}^{h}\left(\tilde{p}, r, \underline{z}, \bar{z}, \tilde{w}^{h}\right)=\left\{\left(x^{h}, \tilde{b}^{h}\right) \in X^{h} \times \mathbb{R}^{S} \mid\right. & \tilde{b}^{h} \in \beta^{h}\left(\tilde{p}, x^{h}\right), \\
& \underline{z} \leq x^{h}-e^{h} \leq \bar{z}, \\
& \left.\tilde{p} x^{h}+r \tilde{b}^{h} \leq \tilde{p} e^{h}+\tilde{w}^{h}\right\} .
\end{aligned}
$$

The two differences between $\gamma^{h}\left(p, q, r, \underline{z}, \bar{z}, v^{h}\right)$ and $\hat{\gamma}^{h}\left(p, q, r, \underline{z}, \bar{z}, v^{h}\right)$ are the inequality rather than the equality in the budget constraint, and the use of real numbers rather than extended real numbers for rationing schemes. The existence proofs are such that the use of extended real numbers for rationing schemes can be avoided. The inequality in the budget constraint is introduced to ensure that $\hat{\gamma}^{h}$ is non-empty valued. Indeed, by A4, it holds that $0 \in \beta^{h}\left(p, e^{h}\right)$. Let $a^{h}$ solve the recursive system of equations (4.2) for $b^{h}=0$. Then we have $\left(e^{h}, a^{h}, 0\right) \in \hat{\gamma}^{h}\left(p, q, r, \underline{z}, \bar{z}, v^{h}\right)$. The correspondence $\gamma^{h}$ on the other hand, can be empty valued. Empty values for $\gamma^{h}\left(p, q, r, \underline{z}, \bar{z}, v^{h}\right)$ could for instance occur when $\bar{z}=0$ and $v^{h}$ is strictly positive.

The correspondence $\tilde{\gamma}^{h}$ is a reformulation of the correspondence $\hat{\gamma}^{h}$ in present-value terms and omits the determination of the $a^{h}$ variables. The proofs of equilibrium existence 
require continuity properties of both the correspondences $\hat{\gamma}^{h}$ and $\tilde{\gamma}^{h}$, which extend similar continuity properties for non-monetary economies provided in Drèze (1975) and Herings (1996a).

LEMMA 4.1 The correspondence $\tilde{\gamma}^{h}$ is lower hemi-continuous and closed at any $\left(\tilde{p}, r, \underline{z}, \bar{z}, \tilde{w}^{h}\right) \in \mathbb{R}_{+}^{L S} \times \mathbb{R}_{+}^{S} \times-\mathbb{R}_{+}^{L S} \times \mathbb{R}_{+}^{L S} \times \mathbb{R}_{+}$satisfying $\tilde{p} \underline{z}<0$ or $\tilde{w}^{h}>0$.

Proof: Let $\left(\tilde{p}_{n}, r_{n}, \underline{z}_{n}, \bar{z}_{n}, \tilde{w}_{n}^{h}\right)_{n \in \mathbb{N}}$ be a sequence of points in $\mathbb{R}_{+}^{L S} \times \mathbb{R}_{+}^{S} \times-\mathbb{R}_{+}^{L S} \times \mathbb{R}_{+}^{L S} \times$ $\mathbb{R}_{+}$converging to $\left(\tilde{p}, r, \underline{z}, \bar{z}, \tilde{w}^{h}\right)$. Let $\left(x^{h}, \tilde{b}^{h}\right)$ be an element of $\tilde{\gamma}^{h}\left(\tilde{p}, r, \underline{z}, \bar{z}, \tilde{w}^{h}\right)$. The correspondence $\tilde{\gamma}^{h}$ is lower hemi-continuous at $\left(\tilde{p}, r, \underline{z}, \bar{z}, \tilde{w}^{h}\right)$ if there is a sequence $\left(x_{n}^{h}, \tilde{b}_{n}^{h}\right)_{n \in \mathbb{N}}$ such that $\left(x_{n}^{h}, \tilde{b}_{n}^{h}\right) \in \tilde{\gamma}^{h}\left(\tilde{p}_{n}, r_{n}, \underline{z}_{n}, \bar{z}_{n}, \tilde{w}_{n}^{h}\right)$ and $\left(x_{n}^{h}, \tilde{b}_{n}^{h}\right) \rightarrow\left(x^{h}, \tilde{b}^{h}\right)$.

We consider two cases, 1. $\tilde{p} x^{h}+r \tilde{b}^{h}<\tilde{p} e^{h}+\tilde{w}^{h}$ and 2. $\tilde{p} x^{h}+r \tilde{b}^{h}=\tilde{p} e^{h}+\tilde{w}^{h}$ and $[\tilde{p} \underline{z}<0$ or $\left.\tilde{w}^{h}>0\right]$.

Case 1. $\tilde{p} x^{h}+r \tilde{b}^{h}<\tilde{p} e^{h}+\tilde{w}^{h}$.

We define the sets $L^{+}=\left\{\ell s_{t} \in L \times S \mid x_{\ell s_{t}}^{h}>e_{\ell s_{t}}^{h}\right\}, L^{0}=\left\{\ell s_{t} \in L \times S \mid x_{\ell s_{t}}^{h}=e_{\ell s_{t}}^{h}\right\}$, and $L^{-}=\left\{\ell s_{t} \in L \times S \mid x_{\ell s_{t}}^{h}<e_{\ell s_{t}}^{h}\right\}$. For $n \in \mathbb{N}$, for $\ell s_{t} \in L^{-}$, let $\lambda_{\ell s_{t}, n}^{h}=\underline{z}_{\ell s_{t}, n} /\left(x_{\ell s_{t}}^{h}-e_{\ell s_{t}}^{h}\right)$, then $\lambda_{\ell s_{t}, n}^{h} \geq 0$ since $x_{\ell s_{t}}^{h}-e_{\ell s_{t}}^{h}<0$ and $\underline{z}_{\ell_{s_{t}, n}} \leq 0$. For $n \in \mathbb{N}$, for $\ell s_{t} \in L^{+}$, let $\lambda_{\ell s_{t}, n}^{h}=$ $\bar{z}_{\ell s_{t}, n} /\left(x_{\ell s_{t}}^{h}-e_{\ell s_{t}}^{h}\right)$, then $\lambda_{\ell s_{t}, n}^{h} \geq 0$ since $x_{\ell s_{t}}^{h}-e_{\ell s_{t}}^{h}>0$ and $\bar{z}_{\ell_{s_{t}, n}} \geq 0$. Finally, let $\lambda_{n}^{h}=$ $\min \left(\left\{\lambda_{\ell s_{t}, n}^{h} \mid \ell s_{t} \in L^{-} \cup L^{+}\right\} \cup\{1\}\right)$. Clearly, $0 \leq \lambda_{n}^{h} \leq 1$. We define $x_{n}^{h}=e^{h}+\lambda_{n}^{h}\left(x^{h}-e^{h}\right)$. Since $x^{h}, e^{h} \in X^{h}$ and by the convexity of $X^{h}$ it holds that $x_{n}^{h} \in X^{h}$. Moreover,

$$
\begin{array}{llrl}
x_{\ell s_{t}, n}^{h}-e_{\ell s_{t}}^{h}=\lambda_{n}^{h}\left(x_{\ell s_{t}}^{h}-e_{\ell s_{t}}^{h}\right) \geq \lambda_{\ell s_{t}, n}^{h}\left(x_{\ell s_{t}}^{h}-e_{\ell s_{t}}^{h}\right)=\underline{z}_{\ell s_{t}, n}, & & \ell s_{t} \in L^{-}, \\
x_{\ell s_{t}, n}^{h}-e_{\ell s_{t}}^{h}=\lambda_{n}^{h}\left(x_{\ell s_{t}}^{h}-e_{\ell s_{t}}^{h}\right) \leq 0 \leq \bar{z}_{\ell s_{t}, n}, & & \ell s_{t} \in L^{-}, \\
x_{\ell s_{t}, n}^{h}-e_{\ell s_{t}}^{h}=\lambda_{n}^{h}\left(x_{\ell s_{t}}^{h}-e_{\ell s_{t}}^{h}\right)=0 \text { and so } \underline{z}_{\ell s_{t}, n} \leq x_{\ell s_{t}, n}^{h}-e_{\ell s_{t}}^{h} \leq \bar{z}_{\ell s_{t}, n}, & \ell s_{t} \in L^{0}, \\
x_{\ell s_{t}, n}^{h}-e_{\ell s_{t}}^{h}=\lambda_{n}^{h}\left(x_{\ell s_{t}}^{h}-e_{\ell s_{t}}^{h}\right) \leq \lambda_{\ell s_{t}, n}^{h}\left(x_{\ell s_{t}}^{h}-e_{\ell s_{t}}^{h}\right)=\bar{z}_{\ell s_{t}, n}, & & \ell s_{t} \in L^{+}, \\
x_{\ell s_{t}, n}^{h}-e_{\ell s_{t}}^{h}=\lambda_{n}^{h}\left(x_{\ell s_{t}}^{h}-e_{\ell s_{t}}^{h}\right) \geq 0 \geq \underline{z}_{\ell s_{t}, n}, & & \ell s_{t} \in L^{+} .
\end{array}
$$

Further,

$$
\begin{aligned}
& \lambda_{\ell s_{t}, n}^{h}=\frac{\underline{z}_{\ell s_{t}, n}}{x_{\ell s_{t}}^{h}-e_{\ell s_{t}}^{h}} \rightarrow \frac{\underline{z}_{\ell s_{t}}}{x_{\ell s_{t}}^{h}-e_{\ell s_{t}}^{h}} \geq \frac{x_{\ell s_{t}}^{h}-e_{\ell s_{t}}^{h}}{x_{\ell s_{t}}^{h}-e_{\ell s_{t}}^{h}}=1, \quad \ell s_{t} \in L^{-}, \\
& \lambda_{\ell s_{t}, n}^{h}=\frac{\bar{z}_{\ell s_{t}, n}}{x_{\ell s_{t}}^{h}-e_{\ell s_{t}}^{h}} \rightarrow \frac{\bar{z}_{\ell s_{t}}}{x_{\ell s_{t}}^{h}-e_{\ell s_{t}}^{h}} \geq \frac{x_{\ell s_{t}}^{h}-e_{\ell s_{t}}^{h}}{x_{\ell s_{t}}^{h}-e_{\ell s_{t}}^{h}}=1, \quad \ell s_{t} \in L^{+} .
\end{aligned}
$$

So $\lambda_{n}^{h} \rightarrow 1$ and therefore $x_{n}^{h}=e^{h}+\lambda_{n}^{h}\left(x^{h}-e^{h}\right) \rightarrow e^{h}+x^{h}-e^{h}=x^{h}$.

Since $\beta^{h}$ is lower hemi-continuous, there exists a sequence $\left(\tilde{b}_{n}^{h}\right)_{n \in \mathbb{N}}$ such that $\tilde{b}_{n}^{h} \in$ $\beta^{h}\left(\tilde{p}_{n}, x_{n}^{h}\right)$ and $\tilde{b}_{n}^{h} \rightarrow \tilde{b}^{h}$. Moreover, $\tilde{p}_{n} x_{n}^{h}+r_{n} \tilde{b}_{n}^{h}-\tilde{p}_{n} e^{h}-\tilde{w}_{n}^{h} \rightarrow \tilde{p} x^{h}+r \tilde{b}^{h}-\tilde{p} e^{h}-\tilde{w}^{h}<0$. Therefore, for $n$ sufficiently large, $\tilde{p}_{n} x_{n}^{h}+r_{n} \tilde{b}_{n}^{h}-\tilde{p}_{n} e^{h}-\tilde{w}_{n}^{h}<0$, so $\left(x_{n}^{h}, \tilde{b}_{n}^{h}\right) \in \tilde{\gamma}^{h}\left(\tilde{p}_{n}, r_{n}, \underline{z}_{n}, \bar{z}_{n}, \tilde{w}_{n}^{h}\right)$ and lower hemi-continuity of $\tilde{\gamma}^{h}$ follows.

Case 2. $\tilde{p} x^{h}+r \tilde{b}^{h}=\tilde{p} e^{h}+\tilde{w}^{h}$ and $\left[\tilde{p} \underline{z}<0\right.$ or $\left.\tilde{w}^{h}>0\right]$. 
Let $\alpha \in(0,1]$ be such that $e^{h}+\alpha \underline{z}_{n} \in X^{h}$ for all $n$ sufficiently large. Such an $\alpha$ exists since $e^{h} \in \operatorname{int}\left(X^{h}\right)$, so $\alpha$ can be chosen such that $e^{h}+\alpha \underline{z} \in \operatorname{int}\left(X^{h}\right)$, and $\underline{z}_{n} \rightarrow \underline{z}$. We define $\hat{e}_{n}^{h}=e^{h}+\alpha \underline{z}_{n}$. For $n$ sufficiently large, $\hat{e}_{n}^{h}$ has the following properties,

$$
\hat{e}_{n}^{h} \in X^{h}, \underline{z}_{n} \leq \alpha \underline{z}_{n}=\hat{e}_{n}^{h}-e^{h} \leq 0 \leq \bar{z}_{n} .
$$

Since $\hat{e}_{n}^{h} \leq e^{h}$ it holds by A4.5 that $0 \in \beta^{h}\left(\tilde{p}_{n}, \hat{e}_{n}^{h}\right)$. For $n$ sufficiently large we have

$$
\tilde{p}_{n} \hat{e}_{n}^{h}+r_{n} 0<\tilde{p}_{n} e^{h}+\tilde{w}_{n}^{h} .
$$

The strict inequality follows since, for $n$ sufficiently large, $\tilde{p} \underline{z}<0$ implies $\tilde{p}_{n} \hat{e}_{n}^{h}<\tilde{p}_{n} e^{h}$ and $r_{n} 0 \leq \tilde{w}_{n}^{h}$, whereas $\tilde{w}^{h}>0$ implies $\tilde{p}_{n} \hat{e}_{n}^{h} \leq \tilde{p}_{n} e^{h}$ and $r_{n} 0<\tilde{w}_{n}^{h}$. Moreover, when we define $\hat{e}^{h}=e^{h}+\alpha \underline{z}$, then we have that $\hat{e}_{n}^{h} \rightarrow \hat{e}^{h}$,

$$
\hat{e}^{h} \in X^{h}, \underline{z} \leq \alpha \underline{z}=\hat{e}^{h}-e^{h} \leq 0 \leq \bar{z} \text {, and } \tilde{p} \hat{e}^{h}+r 0<\tilde{p} e^{h}+\tilde{w}^{h} .
$$

Consider the sequence $\left(x_{n}^{h}, \tilde{b}_{n}^{h}\right)_{n \in \mathbb{N}}$ as defined in Case 1. It may be assumed that the elements of this sequence satisfy

$$
x_{n}^{h} \in X^{h}, \underline{z}_{n} \leq x_{n}^{h}-e^{h} \leq \bar{z}_{n}, x_{n}^{h} \rightarrow x^{h} \text {, and } \tilde{b}_{n}^{h} \in \beta^{h}\left(\tilde{p}_{n}, x_{n}^{h}\right) .
$$

If $\tilde{p}_{n} x_{n}^{h}+r_{n} \tilde{b}_{n}^{h}>\tilde{p}_{n} e^{h}+\tilde{w}_{n}^{h}$, then define $\mu_{n}^{h}$ by

$$
\mu_{n}^{h}=\frac{\tilde{p}_{n} e^{h}+\tilde{w}_{n}^{h}-\tilde{p}_{n} \hat{e}_{n}^{h}}{\tilde{p}_{n} x_{n}^{h}+r_{n} \tilde{b}_{n}^{h}-\tilde{p}_{n} \hat{e}_{n}^{h}}
$$

and if $\tilde{p}_{n} x_{n}^{h}+r_{n} \tilde{b}_{n}^{h} \leq \tilde{p}_{n} e^{h}+\tilde{w}_{n}^{h}$, then define $\mu_{n}^{h}=1$. It holds that $0 \leq \mu_{n}^{h} \leq 1$. We define $\hat{x}_{n}^{h}=\hat{e}_{n}^{h}+\mu_{n}^{h}\left(x_{n}^{h}-\hat{e}_{n}^{h}\right)$ and $\hat{b}_{n}^{h}=\mu_{n}^{h} \tilde{b}_{n}^{h}$. Using the convexity of $X^{h}$, it holds that $\hat{x}^{h} \in X^{h}$.

By (4.3) and (4.4),

$$
\begin{aligned}
& \hat{x}_{n}^{h}-e^{h}=\mu_{n}^{h}\left(x_{n}^{h}-e^{h}\right)+\left(1-\mu_{n}^{h}\right)\left(\hat{e}_{n}^{h}-e^{h}\right) \geq \underline{z}_{n}, \\
& \hat{x}_{n}^{h}-e^{h}=\mu_{n}^{h}\left(x_{n}^{h}-e^{h}\right)+\left(1-\mu_{n}^{h}\right)\left(\hat{e}_{n}^{h}-e^{h}\right) \leq \bar{z}_{n} .
\end{aligned}
$$

If $\tilde{p}_{n} x_{n}^{h}+r_{n} \tilde{b}_{n}^{h}>\tilde{p}_{n} e^{h}+\tilde{w}_{n}^{h}$, then by (4.5)

$$
\begin{aligned}
\tilde{p}_{n} \hat{x}_{n}^{h}+r_{n} \hat{b}_{n}^{h} & =\frac{\tilde{p}_{n} e^{h}+\tilde{w}_{n}^{h}-\tilde{p}_{n} \hat{e}_{n}^{h}}{\tilde{p}_{n} x_{n}^{h}+r_{n} \tilde{b}_{n}^{h}-\tilde{p}_{n} \hat{e}_{n}^{h}}\left(\tilde{p}_{n} x_{n}^{h}+r_{n} \tilde{b}_{n}^{h}\right)+\frac{\tilde{p}_{n} x_{n}^{h}+r_{n} \tilde{b}_{n}^{h}-\tilde{p}_{n} e^{h}-\tilde{w}_{n}^{h}}{\tilde{p}_{n} x_{n}^{h}+r_{n} \tilde{b}_{n}^{h}-\tilde{p}_{n} \hat{e}_{n}^{h}} \tilde{p}_{n} \hat{e}_{n}^{h} \\
& =\tilde{p}_{n} e^{h}+\tilde{w}_{n}^{h},
\end{aligned}
$$

and if $\tilde{p}_{n} x_{n}^{h}+r_{n} \hat{b}_{n}^{h} \leq \tilde{p}_{n} e^{h}+\tilde{w}_{n}^{h}$ then because $\mu_{n}^{h}=1$

$$
\tilde{p}_{n} \hat{x}_{n}^{h}+r_{n} \hat{b}_{n}^{h}=\tilde{p}_{n} x_{n}^{h}+r_{n} \tilde{b}_{n}^{h} \leq \tilde{p}_{n} e^{h}+\tilde{w}_{n}^{h} .
$$

Since $\hat{e}_{n}^{h} \leq e^{h}$, it holds that $0 \in \beta^{h}\left(\tilde{p}_{n}, x_{n}^{h}\right)$ by A4.5. By A4.4 it holds that

$$
\hat{b}_{n}^{h}=\mu_{n}^{h} \tilde{b}_{n}^{h}+\left(1-\mu_{n}^{h}\right) 0 \in \beta^{h}\left(\tilde{p}_{n}, \mu_{n}^{h} x_{n}^{h}+\left(1-\mu_{n}^{h}\right) \hat{e}_{n}^{h}\right)=\beta^{h}\left(\tilde{p}_{n}, \hat{x}_{n}^{h}\right) .
$$


It follows that $\left(\hat{x}_{n}^{h}, \hat{b}_{n}^{h}\right) \in \tilde{\gamma}^{h}\left(\tilde{p}_{n}, r_{n}, \underline{z}_{n}, \bar{z}_{n}, \tilde{w}_{n}^{h}\right)$. Using $\left(x_{n}^{h}, \tilde{b}_{n}^{h}\right) \rightarrow\left(x^{h}, \tilde{b}^{h}\right)$ and $\tilde{p} x^{h}+r \tilde{b}^{h}=$ $\tilde{p} e^{h}+\tilde{w}^{h}$,

$$
\frac{\tilde{p}_{n} e^{h}+\tilde{w}_{n}^{h}-\tilde{p}_{n} \hat{e}_{n}^{h}}{\tilde{p}_{n} x_{n}^{h}+r_{n} \tilde{b}_{n}^{h}-\tilde{p}_{n} \hat{e}_{n}^{h}} \rightarrow \frac{\tilde{p} e^{h}+\tilde{w}^{h}-\tilde{p} \hat{e}^{h}}{\tilde{p} x^{h}+r \tilde{b}^{h}-\tilde{p} \hat{e}^{h}}=1
$$

and so $\mu_{n}^{h} \rightarrow 1$. Consequently, $\hat{x}_{n}^{h} \rightarrow \hat{e}^{h}+\left(x^{h}-\hat{e}^{h}\right)=x^{h}$ and $\hat{b}_{n}^{h} \rightarrow \tilde{b}^{h}$. Lower hemi-continuity of $\tilde{\gamma}^{h}$ follows.

Let $\left(\tilde{p}_{n}, r_{n}, \underline{z}_{n}, \bar{z}_{n}, \tilde{w}_{n}^{h}\right)_{n \in \mathbb{N}}$ be a sequence of points in $\mathbb{R}_{+}^{L S} \times \mathbb{R}_{+}^{S} \times-\mathbb{R}_{+}^{L S} \times \mathbb{R}_{+}^{L S} \times \mathbb{R}_{+}$converging to $\left(\tilde{p}, r, \underline{z}, \bar{z}, \tilde{w}^{h}\right)$. For $n \in \mathbb{N}$, let $\left(x_{n}^{h}, \tilde{b}_{n}^{h}\right)$ be an element of $\tilde{\gamma}^{h}\left(\tilde{p}, r, \underline{z}, \bar{z}, \tilde{w}^{h}\right)$ converging to $\left(x^{h}, \tilde{b}^{h}\right)$. The correspondence $\tilde{\gamma}^{h}$ is closed at $\left(\tilde{p}, r, \underline{z}, \bar{z}, \tilde{w}^{h}\right)$ if $\left(x^{h}, \tilde{b}^{h}\right) \in \tilde{\gamma}^{h}\left(\tilde{p}, r, \underline{z}, \bar{z}, \tilde{w}^{h}\right)$. Since $X^{h}$ is closed by A1, it holds that $\left(x^{h}, \tilde{b}^{h}\right) \in X^{h} \times \mathbb{R}^{S}$. Since $\beta^{h}$ is closed by A4.1, we have $\tilde{b}^{h} \in \beta^{h}\left(\tilde{p}, x^{h}\right)$. The usual continuity arguments imply that $\underline{z} \leq x^{h}-e^{h} \leq \bar{z}$, and $\tilde{p} x^{h}+r \tilde{b}^{h} \leq \tilde{p} e^{h}+\tilde{w}^{h}$. It follows that $\left(x^{h}, \tilde{b}^{h}\right) \in \tilde{\gamma}^{h}\left(\tilde{p}, r, \underline{z}, \bar{z}, \tilde{w}^{h}\right)$.

The assumptions in Lemma 4.1 are such that for every household there is a consumption bundle in the budget set which is strictly less expensive than the household's total income. This cheaper point assumption is well-known in general equilibrium theory, see Debreu (1959), and crucial to show lower hemi-continuity of the budget correspondence. A similar assumption is made in Lemma 4.2.

LEMMA 4.2 The correspondence $\hat{\gamma}^{h}$ is lower hemi-continuous and closed at any $\left(p, q, r, \underline{z}, \bar{z}, v^{h}\right) \in \mathbb{R}_{+}^{L S} \times \bar{Q} \times-\mathbb{R}_{+}^{L S} \times \mathbb{R}_{+}^{L S} \times \mathbb{R}_{+}^{S}$ satisfying $\tilde{p} \underline{z}<0$ or $\tilde{q} v^{h}>0$.

Proof: Let $\left(p_{n}, q_{n}, r_{n}, \underline{z}_{n}, \bar{z}_{n}, v_{n}^{h}\right)_{n \in \mathbb{N}}$ be a sequence of points in $\mathbb{R}_{+}^{L S} \times \bar{Q} \times-\mathbb{R}_{+}^{L S} \times$ $\mathbb{R}_{+}^{L S} \times \mathbb{R}_{+}^{S}$ converging to $\left(p, q, r, \underline{z}, \bar{z}, v^{h}\right)$. Let $\left(x^{h}, a^{h}, b^{h}\right)$ be an element of $\hat{\gamma}^{h}\left(p, q, r, \underline{z}, \bar{z}, v^{h}\right)$. The correspondence $\hat{\gamma}^{h}$ is lower hemi-continuous at $\left(p, q, r, \underline{z}, \bar{z}, v^{h}\right)$ if there is a sequence $\left(x_{n}^{h}, a_{n}^{h}, b_{n}^{h}\right)_{n \in \mathbb{N}}$ such that $\left(x_{n}^{h}, a_{n}^{h}, b_{n}^{h}\right) \in \hat{\gamma}^{h}\left(p_{n}, q_{n}, r_{n}, \underline{z}_{n}, \bar{z}_{n}, v_{n}^{h}\right)$ and $\left(x_{n}^{h}, a_{n}^{h}, b_{n}^{h}\right) \rightarrow\left(x^{h}, a^{h}, b^{h}\right)$.

For $s_{t} \in S$, we define $\tilde{q}_{s_{t}}=q_{s_{t}}^{0} /\left(1+r_{s_{t}}\right), \tilde{p}_{s_{t}}=\tilde{q}_{s_{t}} p_{s_{t}}, \tilde{b}_{s_{t}}^{h}=\tilde{q}_{s_{t}} b_{s_{t}}^{h}, \tilde{v}_{s_{t}}^{h}=\tilde{q}_{s_{t}} v_{s_{t}}^{h}$, and $\tilde{w}_{s_{t}}^{h}=\sum_{s_{t} \in S} \tilde{v}_{s_{t}}^{h}$.

We consider two cases, 1. $\tilde{p} x^{h}+r \tilde{b}^{h}<\tilde{p} e^{h}+\tilde{w}^{h}$ and 2. $\tilde{p} x^{h}+r \tilde{b}^{h}=\tilde{p} e^{h}+\tilde{w}^{h},[\tilde{p} \underline{z}<0$ or $\left.\tilde{w}^{h}>0\right]$.

Case 1. $\tilde{p} x^{h}+r \tilde{b}^{h}<\tilde{p} e^{h}+\tilde{w}^{h}$.

We define the sequence $\left(x_{n}^{h}\right)_{n \in \mathbb{N}}$ as in Case 1 of Lemma 4.1. It holds that $x_{n}^{h} \in X^{h}$, $\underline{z}_{n} \leq x_{n}^{h}-e^{h} \leq \bar{z}_{n}$, and $x_{n}^{h} \rightarrow x^{h}$. Since $\beta^{h}$ is lower hemi-continuous, there exists a sequence $\left(b_{n}^{h}\right)_{n \in \mathbb{N}}$ such that $b_{n}^{h} \in \beta^{h}\left(p_{n}, x_{n}^{h}\right)$ and $b_{n}^{h} \rightarrow b^{h}$. Moreover, $\tilde{p}_{n} x_{n}^{h}+r_{n} \tilde{b}_{n}^{h}-\tilde{p}_{n} e^{h}-\tilde{w}_{n}^{h} \rightarrow \tilde{p} x^{h}+$ $r \tilde{b}^{h}-\tilde{p} e^{h}-\tilde{w}^{h}<0$. Therefore, for $n$ sufficiently large, $\tilde{p}_{n} x_{n}^{h}+r_{n} \tilde{b}_{n}^{h}-\tilde{p}_{n} e^{h}-\tilde{w}_{n}^{h}<0$. We define $a_{n}^{h}$ by means of the recursive system of equations (4.2) as determined by $\left(p_{n}, q_{n}, r_{n}, v_{n}^{h}\right)$ and $\left(x_{n}^{h}, b_{n}^{h}\right)$. It is straightforward to demonstrate that $a_{n}^{h} \rightarrow a^{h}$. Clearly, for $n$ sufficiently large, 
$\left(x_{n}^{h}, a_{n}^{h}, b_{n}^{h}\right) \in \hat{\gamma}^{h}\left(p_{n}, q_{n}, r_{n}, \underline{z}_{n}, \bar{z}_{n}, v_{n}^{h}\right)$ and lower hemi-continuity of $\hat{\gamma}^{h}$ follows.

Case 2. $\tilde{p} x^{h}+\tilde{r} b^{h}=\tilde{p} e^{h}+\tilde{w}^{h},\left[\tilde{p} \underline{z}<0\right.$ or $\left.\tilde{w}^{h}>0\right]$.

Consider the sequence $\left(x_{n}^{h}, a_{n}^{h}, b_{n}^{h}\right)_{n \in \mathbb{N}}$ as defined in Case 1 and the sequence $\left(\hat{e}_{n}^{h}\right)_{n \in \mathbb{N}}$ as defined in Case 2 of Lemma 4.1. For $n \in \mathbb{N}$, for $s_{t} \in S$, we define $\tilde{b}_{s_{t}, n}^{h}=\tilde{q}_{s_{t}, n} b_{s_{t}, n}^{h}$. If $\tilde{p}_{n} x_{n}^{h}+r_{n} \tilde{b}_{n}^{h}>\tilde{p}_{n} e^{h}+\tilde{w}_{n}^{h}$, then define $\mu_{n}^{h}$ by

$$
\mu_{n}^{h}=\frac{\tilde{p}_{n} e^{h}+\tilde{w}_{n}^{h}-\tilde{p}_{n} \hat{e}_{n}^{h}}{\tilde{p}_{n} x_{n}^{h}+r_{n} \tilde{b}_{n}^{h}-\tilde{p}_{n} \hat{e}_{n}^{h}}
$$

and if $\tilde{p}_{n} x_{n}^{h}+r_{n} \tilde{b}_{n}^{h} \leq \tilde{p}_{n} e^{h}+\tilde{w}_{n}^{h}$, then define $\mu_{n}^{h}=1$. Next, we define $\hat{x}_{n}^{h}=\hat{e}_{n}^{h}+\mu_{n}^{h}\left(x_{n}^{h}-\hat{e}_{n}^{h}\right)$ and $\hat{b}_{n}^{h}=\mu_{n}^{h} b_{n}^{h}$. We define $\hat{a}_{n}^{h}$ by means of the recursive system of equations (4.2) as determined by $\left(p_{n}, q_{n}, r_{n}, v_{n}^{h}\right)$ and $\left(\hat{x}_{n}^{h}, \hat{b}_{n}^{h}\right)$.

By exactly the same arguments as in the proof of Case 1 of Lemma 4.1 it follows that $\left(\hat{x}_{n}^{h}, \hat{a}_{n}^{h}, \hat{b}_{n}^{h}\right) \in \hat{\gamma}^{h}\left(p_{n}, q_{n}, r_{n}, \underline{z}_{n}, \bar{z}_{n}, v_{n}^{h}\right)$ and $\left(\hat{x}_{n}^{h}, \hat{a}_{n}^{h}, \hat{b}_{n}^{h}\right) \rightarrow\left(x^{h}, a^{h}, b^{h}\right)$. Lower hemi-continuity of $\hat{\gamma}^{h}$ follows.

Let $\left(p_{n}, q_{n}, r_{n}, \underline{z}_{n}, \bar{z}_{n}, v_{n}^{h}\right)_{n \in \mathbb{N}}$ be a sequence of points in $\mathbb{R}_{+}^{L S} \times \bar{Q} \times-\mathbb{R}_{+}^{L S} \times \mathbb{R}_{+}^{L S} \times \mathbb{R}_{+}^{S}$ converging to $\left(p, q, r, \underline{z}, \bar{z}, v^{h}\right)$. For $n \in \mathbb{N}$, let $\left(x_{n}^{h}, a_{n}^{h}, b_{n}^{h}\right)$ be an element of $\hat{\gamma}^{h}\left(p, q, r, \underline{z}, \bar{z}, v^{h}\right)$ converging to $\left(x^{h}, a^{h}, b^{h}\right)$. The correspondence $\hat{\gamma}^{h}$ is closed at $\left(p, q, r, \underline{z}, \bar{z}, v^{h}\right)$ if $\left(x^{h}, a^{h}, b^{h}\right) \in$ $\hat{\gamma}^{h}\left(p, q, r, \underline{z}, \bar{z}, v^{h}\right)$. Since $X^{h}$ is closed by A1, it holds that $\left(x^{h}, a^{h}, b^{h}\right) \in X^{h} \times \mathbb{R}^{S^{+}} \times \mathbb{R}^{S}$. Since $\beta^{h}$ is closed by A4.1, we have $b^{h} \in \beta^{h}(p, x)$. The usual continuity arguments imply that

$$
\begin{array}{ll}
\underline{z} \leq x^{h}-e^{h} \leq \bar{z}, & \\
\tilde{p} x^{h}+\tilde{r} b^{h} \leq \tilde{p} e^{h}+\tilde{q} v^{h}, & \\
a_{s_{T+1}}^{h}=p_{s_{T+1}}^{-}\left(x_{s_{T+1}^{-}}^{h}-e_{s_{T+1}^{-}}^{h}\right)+r_{s_{T+1}^{-}} b_{s_{T+1}^{-}}^{h}-v_{s_{T+1}^{-}}^{h}, & s_{T+1} \in S_{T+1}, \\
a_{s_{t}}^{h}=p_{s_{t}}^{-}\left(x_{s_{t}^{-}}^{h}-e_{s_{t}^{-}}^{h}\right)+r_{s_{t}^{-}} b_{s_{t}^{-}}^{h}-v_{s_{t}^{-}}^{h}+\sum_{s_{t+1} \in s_{t}^{+}} q_{s_{t+1}} a_{s_{t+1}}^{h}, & s_{t} \in S \backslash\left\{s_{0}\right\} .
\end{array}
$$

It follows that $\left(x^{h}, a^{h}, b^{h}\right) \in \hat{\gamma}^{h}\left(p, q, r, \underline{z}, \bar{z}, v^{h}\right)$.

\section{$5 \quad$ Parametrized Prices and Rationing Schemes}

We will not only establish that sticky price equilibria exist, but also that there is an abundance of such equilibria. In fact, we will argue that the set of sticky price equilibria is $\left|S_{T}\right|$-dimensional. To prove this result, it is convenient to choose a suitable parametrization of price and rationing variables. For $\ell s_{t} \in L \times S$, we define the set of parameters $R_{\ell s_{t}}$ by

$$
\begin{aligned}
& R_{\ell_{s_{t}}}=\mathbb{R}_{+}, \quad \text { if } s_{t} \in N_{\ell}^{\mathrm{a}}, \\
& R_{\ell s_{t}}=[0,1], \quad \text { if } s_{t} \in N_{\ell}^{\mathrm{s}},
\end{aligned}
$$

with typical element $\rho_{\ell s_{t}}$. Next, we define $R=\prod_{\ell_{t} \in L \times S} R_{\ell_{s_{t}}}$ with typical element $\rho$. 
When $s_{t} \in N_{\ell}^{\mathrm{a}}$, supply and demand are equilibrated by the price $p_{\ell s_{t}} \in \mathbb{R}_{+}$. We parametrize this price by the variable $\rho_{\ell s_{t}} \in \mathbb{R}_{+}$and set $p_{\ell s_{t}}(\rho)=\rho_{\ell s_{t}}$.

At $s_{t}$ in $N_{\ell}^{\mathrm{s}}$, the market for commodity $\ell$ is equilibrated by one-sided rationing. We will parametrize the relevant rationing schemes by a single parameter $\rho_{\ell s_{t}} \in[0,1]$. We define $\underline{z}_{\ell_{s t}}(\rho)$ and $\bar{z}_{\ell s_{t}}(\rho)$ in such a way that the following properties hold: $\underline{z}_{\ell_{s t}}(\rho)=0$ if $\rho_{\ell s_{t}}=0$, at equilibrium $\rho$ may lead to supply rationing in market for commodity $\ell s_{t}$ only if $\rho_{\ell s_{t}}<1 / 2$, but is irrelevant for supply rationing when $\rho_{\ell s_{t}} \geq 1 / 2$. Similarly, $\bar{z}_{\ell s_{t}}(\rho)=0$ if $\rho_{\ell s_{t}}=1$, at equilibrium $\rho_{\ell s_{t}}$ may lead to demand rationing in market for commodity $\ell s_{t}$ only if $\rho_{\ell s_{t}}>1 / 2$, but is irrelevant for demand rationing when $\rho_{\ell s_{t}} \leq 1 / 2$. The rationing scheme on supply induced by $\rho$ is more negative, so less restrictive for choice, when $\rho_{\ell s_{t}}$ increases; the induced rationing scheme on demand is less positive, so more restrictive when $\rho_{\ell s_{t}}$ increases. In this way $\rho_{\ell s_{t}}$ clears markets by means of rationing in a way analogously to a price variable. Low values of $\rho_{\ell_{s t}}$ tend to induce excess demand and high values of $\rho_{\ell s_{t}}$ tend to induce excess supply, completely analogous to the response of excess demand to the price of a commodity.

We now make the parametrization explicit. Since consumption sets are bounded from below, the market clearing conditions imply that the set of attainable allocations of consumption bundles, the set of $x \in \prod_{h \in H} X^{h}$ such that $\sum_{h \in H} x^{h}=\sum_{h \in H} e^{h}$, is bounded. Let $\bar{c} \in \mathbb{R}_{+}^{L S}$ be such that at every attainable allocation the excess consumption $x_{\ell s_{t}}^{h}-e_{\ell s_{t}}^{h}$ of every household $h$ is strictly less than $\bar{c}_{\ell s_{t}}$ and strictly more than $-\bar{c}_{\ell s_{t}}$. Moreover, we choose $\bar{c} \geq \sum_{h \in H}\left(e^{h}-\underline{x}^{h}\right)$, where $\underline{x}^{h}$ is a lower bound for $X^{h}$, which implies that consumption equal to $\bar{c}_{\ell_{t}}$ by a single household is not compatible with feasibility of the allocation.

We define, for $\ell \in L$, for $s_{t} \in N_{\ell}^{\mathrm{a}}$,

$$
\begin{aligned}
p_{\ell s_{t}}(\rho) & =\rho_{\ell s_{t}}, \\
\underline{z}_{\ell s_{t}}(\rho) & =-\bar{c}_{\ell s_{t}}, \\
\bar{z}_{\ell s_{t}}(\rho) & =\bar{c}_{\ell s_{t}},
\end{aligned}
$$

for $s_{t} \in N_{\ell}^{\mathrm{s}}$,

$$
\begin{aligned}
p_{\ell s_{t}}(\rho) & =p_{\ell s_{t}^{-}}(\rho), \\
\underline{z}_{\ell s_{t}}(\rho) & =\max \left\{-2 \bar{c}_{\ell s_{t}} \rho_{\ell s_{t}},-\bar{c}_{\ell s_{t}}\right\}, \\
\bar{z}_{\ell s_{t}}(\rho) & =\min \left\{2 \bar{c}_{\ell s_{t}}-2 \bar{c}_{\ell s_{t}} \rho_{\ell s_{t}}, \bar{c}_{\ell s_{t}}\right\} .
\end{aligned}
$$

In case $s_{0} \in N_{\ell}^{\mathrm{s}}$, the above definition should be read as requiring $p_{\ell s_{0}^{-}}(\rho)=p_{\ell s_{-1}}$.

Notice that this recursive definition implies $p_{\ell s_{t^{\prime}}}(\rho)=\rho_{\ell s_{t}\left(s_{t^{\prime}}\right)}$, where $\ell s_{t^{\prime}}$ is a commodity with a sticky price, $t$ is the date where this price is set, and $s_{t}\left(s_{t^{\prime}}\right)$ the prevailing date-event. In case $t=-1$ it holds that $p_{\ell s_{t^{\prime}}}(\rho)=p_{\ell s_{-1}}$. An important observation is that for each commodity $\ell s_{t}$ there is exactly one instrument, parametrized by $\rho_{\ell s_{t}}$, to clear its market, 
either a price, or a rationing scheme on supply, or a rationing scheme on demand.

Definition 5.1 A parametrized sticky price equilibrium for the economy $\mathcal{E}$ is $\left(\rho^{*}, q^{*}, v^{*}, x^{*}, a^{*}, b^{*}\right)$ in $R \times \mathbb{R}^{S^{+}} \times \mathbb{R}^{H S} \times \mathbb{R}^{H L S} \times \mathbb{R}^{H S^{+}} \times \mathbb{R}^{H S}$ such that

(a) for $h \in H,\left(x^{* h}, a^{* h}, b^{* h}\right)$ is $\preceq^{h}$-maximal on $\gamma^{h}\left(p\left(\rho^{*}\right), q^{*}, r, \underline{z}\left(\rho^{*}\right), \bar{z}\left(\rho^{*}\right), v^{* h}\right)$,

(b) commodity markets clear, $\sum_{h \in H} x^{* h}=\sum_{h \in H} e^{h}$,

(c) Arrow security markets clear, $\sum_{h \in H} a^{* h}=0$,

(d) the no-arbitrage conditions hold, for $s_{t} \in S, \sum_{s_{t+1} \in s_{t}^{+}} q_{s_{t+1}}^{*}=1 /\left(1+r_{s_{t}}\right)$,

(e) for $h \in H$, for $s_{t} \in S$, dividends satisfy $v_{s_{t}}^{* h}=\theta^{h} r_{s_{t}} \sum_{h \in H} b_{s_{t}}^{* h}$.

The notion of parametrized sticky price equilibrium is more convenient than the one of sticky price equilibrium for a number of reasons. The number of free variables in a parametrized sticky price equilibrium is less than the number in a sticky price equilibrium and is equal to the number of market clearing conditions. None of the equilibrium conditions in a parametrized sticky price equilibrium involves conditionals. All the variables in a parametrized sticky price equilibrium are real numbers; extended real numbers are avoided.

The next result shows that a parametrized sticky price equilibrium induces a sticky price equilibrium in a straightforward way. In fact, all that we need to do is to replace supply rationing schemes that are equal to the lower bound $-\bar{c}_{\ell s_{t}}$ by $-\infty$ and demand rationing schemes equal to the upper bound $\bar{c}_{\ell_{s}}$ by $+\infty$. To this end, we define $\underline{z}_{\ell s_{t}}^{\infty}(\rho)=\underline{z}_{\ell s_{t}}(\rho)$ if $\underline{z}_{\ell s_{t}}(\rho)>-\bar{c}_{\ell s_{t}}$ and $\underline{z}_{\ell s_{t}}^{\infty}(\rho)=-\infty$ if $\underline{z}_{\ell s_{t}}(\rho)=-\bar{c}_{\ell s_{t}}$, and $\bar{z}_{\ell s_{t}}^{\infty}(\rho)=\bar{z}_{\ell s_{t}}(\rho)$ if $\bar{z}_{\ell s_{t}}(\rho)<\bar{c}_{\ell s_{t}}$ and $\bar{z}_{\ell s_{t}}^{\infty}(\rho)=+\infty$ if $\bar{z}_{\ell s_{t}}(\rho)=\bar{c}_{\ell s_{t}}$.

THEOREM 5.2 If $\left(\rho^{*}, q^{*}, v^{*}, x^{*}, a^{*}, b^{*}\right)$ is a parametrized sticky price equilibrium, then $\left(p\left(\rho^{*}\right), q^{*}, \underline{z}^{\infty}\left(\rho^{*}\right), \bar{z}^{\infty}\left(\rho^{*}\right), v^{*}, x^{*}, a^{*}, b^{*}\right)$ is a sticky price equilibrium.

Proof: We verify that $\left(p\left(\rho^{*}\right), q^{*}, \underline{z}^{\infty}\left(\rho^{*}\right), \bar{z}^{\infty}\left(\rho^{*}\right), v^{*}, x^{*}, a^{*}, b^{*}\right)$ satisfies Conditions (a)(h) of Definition 2.1.

To show (a), it is sufficient to show that replacing a rationing scheme on supply equal to $-\bar{c}_{\ell s_{t}}$ by $-\infty$ and a rationing scheme on demand equal to $\bar{c}_{\ell s_{t}}$ by $+\infty$ is not going to give opportunities to any household $h$ to acquire $\left(x^{h}, a^{h}, b^{h}\right) \in \gamma^{h}\left(p\left(\rho^{*}\right), q^{*}, \underline{z}^{\infty}\left(\rho^{*}\right), \bar{z}^{\infty}\left(\rho^{*}\right), v^{* h}\right)$ such that $x^{* h} \prec^{h} x^{h}$. Suppose, on the contrary, there is such a household $h$ and a corresponding $\left(x^{h}, a^{h}, b^{h}\right)$. Since $x^{*}$ is an attainable consumption bundle, it holds that $-\bar{c} \ll$ $x^{* h}-e^{h} \ll \bar{c}$. It follows that $-\bar{c} \ll \lambda\left(x^{h}-e^{h}\right)+(1-\lambda)\left(x^{* h}-e^{h}\right) \ll \bar{c}$ for $\lambda$ strictly positive and sufficiently close to zero, and therefore $\underline{z}\left(\rho^{*}\right) \leq \lambda\left(x^{h}-e^{h}\right)+(1-\lambda)\left(x^{* h}-e^{h}\right) \leq \bar{z}\left(\rho^{*}\right)$. 
Since $\gamma^{h}\left(p\left(\rho^{*}\right), q^{*}, r, \underline{z}^{\infty}\left(\rho^{*}\right), \bar{z}^{\infty}\left(\rho^{*}\right), v^{* h}\right)$ is convex, we find that $\left(\lambda x^{h}+(1-\lambda) x^{* h}, \lambda a^{h}+\right.$ $\left.(1-\lambda) a^{* h}, \lambda b^{h}+(1-\lambda) b^{* h}, v^{* h}\right) \in \gamma^{h}\left(p\left(\rho^{*}\right), q^{*}, r, \underline{z}\left(\rho^{*}\right), \bar{z}\left(\rho^{*}\right)\right)$. By convexity of $\preceq^{h}$ we have that $x^{* h} \prec^{h} \lambda x^{h}+(1-\lambda) x^{* h}$, which contradicts that $\left(x^{* h}, a^{* h}, b^{* h}\right)$ is $\preceq^{h}$-maximal on $\gamma^{h}\left(p\left(\rho^{*}\right), q^{*}, r, \underline{z}\left(\rho^{*}\right), \bar{z}\left(\rho^{*}\right), v^{* h}\right)$.

Conditions (b), (c), (d), (e), and (f) hold trivially.

For $s_{t} \in N_{\ell}^{\mathrm{a}}, \underline{z}_{\ell s_{t}}\left(\rho^{*}\right)=-\bar{c}_{\ell s_{t}}$ and $\bar{z}_{\ell s_{t}}\left(\rho^{*}\right)=\bar{c}_{\ell s_{t}}$, so $\underline{z}_{\ell s_{t}}^{\infty}\left(\rho^{*}\right)=-\infty$ and $\bar{z}_{\ell s_{t}}^{\infty}\left(\rho^{*}\right)=+\infty$, and we obtain Condition (g).

By definition, for any value of $\rho \in R$, it is not the case that simultaneously $\underline{z}_{\ell s_{t}}(\rho)>$ $-\bar{c}_{\ell s_{t}}$ and $\bar{z}_{\ell s_{t}}(\rho)<\bar{c}_{\ell s_{t}}$, and therefore it is not the case that simultaneously $\underline{z}_{\ell s_{t}}^{\infty}(\rho)>-\infty$ and $\bar{z}_{\ell s_{t}}^{\infty}(\rho)<+\infty$. This proves that Condition $(\mathrm{h})$ is satisfied.

By Theorem 5.2, if we show that parametrized sticky price equilibria exist, we have shown the existence of sticky price equilibria. Theorem 5.3 shows the converse of Theorem 5.2. Up to irrelevant values of non-binding rationing schemes, all sticky price equilibria are obtained when restricting attention to parametrized sticky price equilibria.

TheOREM 5.3 If $\left(p^{*}, q^{*}, \underline{z}^{*}, \bar{z}^{*}, v^{*}, x^{*}, a^{*}, b^{*}\right)$ is a sticky price equilibrium, then $\left(\rho^{*}, q^{*}, v^{*}, x^{*}, a^{*}, b^{*}\right)$ is a parametrized sticky price equilibrium, where for $s_{t} \in N_{\ell}^{\mathrm{a}}$,

$$
\rho_{\ell s_{t}}^{*}=p_{\ell s_{t}}^{*}
$$

and for $s_{t} \in N_{\ell}^{\mathrm{s}}$,

$$
\begin{array}{ll}
\text { if } \underline{z}_{\ell s_{t}}^{*}<-\bar{c}_{\ell s_{t}} \text { and } \bar{z}_{\ell s_{t}}^{*}>\bar{c}_{\ell s_{t}}, & \text { then } \rho_{\ell s_{t}}^{*}=1 / 2, \\
\text { if } \underline{z}_{\ell s_{t}}^{*} \geq-\bar{c}_{\ell s_{t}}, & \text { then } \rho_{\ell s_{t}}^{*}=\underline{z}_{\ell s_{t}}^{*} /-2 \bar{c}_{\ell s_{t}}, \\
\text { if } \bar{z}_{\ell s_{t}}^{*} \leq \bar{c}_{\ell s_{t}}, & \text { then } \rho_{\ell s_{t}}^{*}=\left(2 \bar{c}_{\ell s_{t}}-\bar{z}_{\ell s_{t}}^{*}\right) / 2 \bar{c}_{\ell s_{t}} .
\end{array}
$$

Proof: We show that $\left(\rho^{*}, q^{*}, v^{*}, x^{*}, a^{*}, b^{*}\right)$ satisfies all the conditions of a parametrized sticky price equilibrium.

Condition (a) follows since

$$
\left(x^{* h}, a^{* h}, b^{* h}\right) \in \gamma^{h}\left(p\left(\rho^{*}\right), q^{*}, r, \underline{z}\left(\rho^{*}\right), \bar{z}\left(\rho^{*}\right), v^{* h}\right) \subset \gamma^{h}\left(p^{*}, q^{*}, r, \underline{z}^{*}, \bar{z}^{*}, v^{* h}\right) .
$$

Conditions (b), (c), (d), and (e) hold trivially.

Theorems 5.2 and 5.3 imply that there is no loss of generality to restrict attention to parametrized sticky price equilibria. 


\section{$6 \quad$ Multiplicity of Sticky Price Equilibria}

We show that the degree of multiplicity of sticky price equilibria is $\left|S_{T}\right|$ by showing that parametrized sticky price equilibria exist that satisfy $\left|S_{T}\right|$ additional restrictions on top of the equilibrium conditions. To make our formulation as simple as possible, we assume that at every terminal date-event $s_{T} \in S_{T}$ there is at least one commodity with a flexible price. We select for each date-event $s_{T}$ one such commodity, denoted by $\ell\left(s_{T}\right) s_{T}$, and define the set of those commodities by $L^{*}=\left\{\ell\left(s_{T}\right) s_{T} \mid s_{T} \in S_{T}\right\}$. The $\left|S_{T}\right|$ additional restrictions are formulated by choosing an arbitrary vector $\alpha \in \mathbb{R}_{++}^{S_{T}}$ and imposing, for $s_{T} \in S_{T}, \rho_{\ell\left(s_{T}\right) s_{T}}=\alpha_{s_{T}}$ on top of the equilibrium conditions. The additional restrictions pin down the nominal prices of $\left|S_{T}\right|$ commodities.

Theorem 6.1 For all $s_{T} \in S_{T}$, assume $s_{T} \in \cup_{\ell \in L} N_{\ell}^{\mathrm{a}}$. Let $L^{*}$ be a set with one flexible price commodity for each terminal date-event. For each choice of $\alpha \in \mathbb{R}_{++}^{S_{T}}$, there is a parametrized sticky price equilibrium $\left(\rho^{*}, q^{*}, v^{*}, x^{*}, a^{*}, b^{*}\right)$ satisfying, for all $\ell s_{T} \in L^{*}, \rho_{\ell_{s_{T}}}^{*}=$ $\alpha_{s_{T}}$.

ProOF:

STEP 1. Compactification.

Fix some $u \geq \max \left\{1, \max _{s_{T} \in S_{T}} \alpha_{s_{T}}, \max _{\ell \in L} p_{\ell s_{-1}}\right\}$. For $\ell s_{t} \in(L \times S) \backslash L^{*}$, we define $\bar{R}_{\ell_{s_{t}}}=$ $R_{\ell_{s_{t}}} \cap[0, u]$ and $\bar{R}=\prod_{\ell_{s_{t}} \in(L \times S) \backslash L^{*}} \bar{R}_{\ell_{s_{t}}} \times\{\alpha\}$. Since $u \geq 1$, the definition of $\bar{R}$ imposes no restrictions on $\rho_{\ell s_{t}}$ when $s_{t} \in N_{\ell}^{\mathrm{s}}$. Since $u \geq \max _{s_{T} \in S_{T}} \alpha_{s_{T}}$ and $u \geq \max _{\ell \in L} p_{\ell_{s_{-1}}}$, we have that $p(\bar{R}) \subset\left[0, u^{S}\right]$, where $u^{S} \in \mathbb{R}^{S}$ is the vector with all components equal to $u$.

For $h \in H$, we define the compact set

$$
\bar{X}^{h}=\left\{x^{h} \in X^{h} \mid x^{h}-e^{h} \leq \bar{c}\right\} .
$$

Choose $f^{h}$ and $\bar{f}^{h}$ as in A4. Since $\left[0, u^{S}\right] \times \bar{X}^{h}$ is compact and $\underline{f}^{h}$ and $\bar{f}^{h}$ are continuous, $\cup_{h \in H} \underline{f}^{h}\left(\left[0, u^{S}\right] \times \bar{X}^{h}\right)$ and $\cup_{h \in H} \bar{f}^{h}\left(\left[0, u^{S}\right] \times \bar{X}^{h}\right)$ are compact. Let $b^{-} \in \mathbb{R}^{S}$ be a lower bound for the former and $b^{+} \in \mathbb{R}^{S}$ be an upper bound for the latter set. We define

$$
\begin{array}{rlrl}
B^{h} & =\left\{b^{h} \in \mathbb{R}^{S} \mid b^{-} \leq b^{h} \leq b^{+}\right\}, & & h \in H, \\
V^{\mathrm{c}} & =\left\{v \in \mathbb{R}_{+}^{S} \mid \forall s_{t} \in S, v_{s_{t}} \leq r_{s_{t}} \sum_{h \in H} b_{s_{t}}^{+}\right\}, & & \\
V^{h} & =V^{\mathrm{c}}, & h \in H .
\end{array}
$$

For $h \in H$, for $s_{t} \in S$, we define $A_{s_{t}}^{h}$ recursively as a compact, convex set containing all $a_{s_{t}^{+}}^{h} \in \mathbb{R}_{t}^{s_{t}^{+}}$such that (4.2) is satisfied for some $p \in\left[0, u^{S}\right],(q, r) \in \bar{Q}, v^{h} \in V^{h}, x^{h} \in \bar{X}^{h}$, $a_{\left(s_{t}^{+}\right)^{+}}^{h^{t}} \in A_{s_{t}^{+}}^{h}$, and $b^{h} \in B^{h}$. Next, we define $A^{h}=\prod_{s_{t} \in S} A_{s_{t}}^{h}$. 
STEP 2. Formulation of the fixed point correspondence.

For $\ell s_{t} \in(L \times S) \backslash L^{*}$, we define

$$
\mu_{\ell s_{t}}(x)=\left\{\bar{\rho}_{\ell s_{t}} \in \bar{R}_{\ell s_{t}} \mid \bar{\rho}_{\ell s_{t}} \sum_{h \in H}\left(x_{\ell s_{t}}^{h}-e_{\ell s_{t}}^{h}\right) \geq \rho_{\ell s_{t}} \sum_{h \in H}\left(x_{\ell s_{t}}^{h}-e_{\ell s_{t}}^{h}\right) \text { for all } \rho_{\ell s_{t}} \in \bar{R}_{\ell s_{t}}\right\} .
$$

We define the correspondence $\mu: \bar{X} \rightarrow \bar{R}$ by setting $\mu(x)=\prod_{\ell s_{t} \in(L \times S) \backslash L^{*}} \mu_{\ell s_{t}}(x) \times\{\alpha\}$.

For $s_{t} \in S$, we define $Q_{s_{t}}=\left\{q_{s_{t}^{+}} \in \mathbb{R}_{+}^{s_{t}^{+}} \mid \sum_{s_{t+1} \in s_{t}^{+}} q_{s_{t+1}}=1 /\left(1+r_{s_{t}}\right)\right\}$ and the correspondence $\nu_{s_{t}}: A_{s_{t}} \rightarrow Q_{s_{t}}$ by

$$
\nu_{s_{t}}\left(a_{s_{t}^{+}}\right)=\left\{\bar{q}_{s_{t}^{+}} \in Q_{s_{t}} \mid \bar{q}_{s_{t}^{+}} \sum_{h \in H} a_{s_{t}^{+}}^{h} \geq q_{s_{t}^{+}} \sum_{h \in H} a_{s_{t}^{+}}^{h}\right\}, \quad a_{s_{t}^{+}} \in A_{s_{t}} .
$$

For $h \in H$, we define the (single-valued) correspondence $o^{h}: B^{h} \rightarrow V^{h}$ by

$$
o_{s_{t}}^{h}\left(b^{h}\right)=\left\{\theta^{h} r_{s_{t}} \sum_{h^{\prime} \in H} b_{s_{t}}^{h^{\prime}}\right\}, \quad s_{t} \in S .
$$

We define the correspondence $\bar{\gamma}^{h}: \bar{R} \times Q \times V^{h} \rightarrow \bar{X}^{h} \times A^{h} \times B^{h}$ by

$$
\bar{\gamma}^{h}\left(\rho, q, v^{h}\right)=\hat{\gamma}^{h}\left(p(\rho), q, r, \underline{z}(\rho), \bar{z}(\rho), v^{h}\right) \cap\left(X^{h} \times A^{h} \times B^{h}\right), \quad\left(\rho, q, v^{h}\right) \in \bar{R} \times Q \times V^{h} .
$$

The construction of the functions $\underline{z}$ and $\bar{z}$ guarantees that any consumption bundle in $\hat{\gamma}^{h}\left(p(\rho), q, r, \underline{z}(\rho), \bar{z}(\rho), v^{h}\right)$ belongs to $\bar{X}^{h}$. The construction of $B^{h}$ ensures that any $\preceq^{h_{-}}$ optimal consumption bundle in $\hat{\gamma}^{h}\left(p(\rho), q, r, \underline{z}(\rho), \bar{z}(\rho), v^{h}\right)$ can be purchased by means of bank loans in $B^{h}$. Finally, the choice of $A^{h}$ is such that any pair consisting of a consumption bundle in $\hat{\gamma}^{h}\left(p(\rho), q, r, \underline{z}(\rho), \bar{z}(\rho), v^{h}\right)$ and a bank loan in $B^{h}$ can be financed by asset market transactions in $A^{h}$.

For all $q \in Q$, there is $s_{T} \in S_{T}$ such that $q_{s_{T}}^{0}>0$ and therefore

$$
\frac{q_{s_{T}}^{0}}{1+r_{s_{T}}} p_{\ell\left(s_{T}\right) s_{T}}(\rho)=\frac{q_{s_{T}}^{0}}{1+r_{s_{T}}} \alpha_{s_{T}}>0
$$

SO

$$
\sum_{s_{t} \in S} \frac{q_{s_{t}}^{0}}{1+r_{s_{t}}} p_{s_{t}}(\rho) \underline{z}_{s_{t}}(\rho) \leq-\frac{q_{s_{T}}^{0}}{1+r_{s_{T}}} \alpha_{s_{T}} \bar{c}_{\ell\left(s_{T}\right) s_{T}}<0,
$$

and it follows by Lemma 4.2 that $\hat{\gamma}^{h}$ is lower hemi-continuous at any $\left(p(\rho), q, r, \underline{z}(\rho), \bar{z}(\rho), v^{h}\right)$ with $\left(\rho, q, v^{h}\right) \in \bar{R} \times Q \times V^{h}$. For all $\left(\rho, q, v^{h}\right) \in \bar{R} \times Q \times V^{h}, \operatorname{int}\left(\hat{\gamma}^{h}\left(p(\rho), q, r, \underline{z}(\rho), \bar{z}(\rho), v^{h}\right)\right) \cap$ $\operatorname{int}\left(X^{h} \times A^{h} \times B^{h}\right) \neq \emptyset$, so it follows from Hildenbrand (1974), Problem 6, p. 35, that $\bar{\gamma}^{h}$ is lower hemi-continuous. Since $\bar{X}^{h} \times A^{h} \times B^{h}$ is compact, and $\hat{\gamma}^{h}$ is closed by Lemma 4.2, we have that $\bar{\gamma}^{h}$ has a closed graph, so is upper hemi-continuous. We define $\delta^{h}\left(\rho, q, v^{h}\right)$ as 
the set of $\preceq^{h}$-maximal elements on $\bar{\gamma}^{h}\left(\rho, q, v^{h}\right)$. An application of the maximum theorem demonstrates that $\delta^{h}$ is an upper hemi-continuous correspondence.

Consider the correspondence $\varphi: R \times Q \times V \times \bar{X} \times A \times B \rightarrow R \times Q \times V \times \bar{X} \times A \times B$ defined by

$$
\varphi(\rho, q, v, x, a, b)=\mu(x) \times \nu(a) \times o(b) \times \delta(\rho, q, v), \quad(\rho, q, v, x, a, b) \in R \times Q \times V \times \bar{X} \times A \times B,
$$

where all correspondences involved in the definition of $\varphi$ are defined as the obvious products.

STEP 3. Existence of a fixed point.

Since the domain of $\varphi$ is a non-empty, compact, and convex set, and $\varphi$ is an upper hemi-continuous convex-valued correspondence, all conditions of Kakutani's fixed point theorem are satisfied. The correspondence $\varphi$ has a fixed point $\left(\rho^{*}, q^{*}, v^{*}, x^{*}, a^{*}, b^{*}\right) \in$ $\mu\left(x^{*}\right) \times \nu\left(a^{*}\right) \times o\left(b^{*}\right) \times \delta\left(\rho^{*}, q^{*}, v^{*}\right)$. We define $p^{*}=p\left(\rho^{*}\right), \underline{z}^{*}=\underline{z}\left(\rho^{*}\right), \bar{z}^{*}=\bar{z}\left(\rho^{*}\right)$, and $z^{*}=x^{*}-e$. Moreover, we define present-value prices $\tilde{q}^{*}, \tilde{p}^{*}, \tilde{b}^{*}, \tilde{v}^{*}$, and $\tilde{w}^{*}$ by setting, for $s_{t} \in S, \tilde{q}_{s_{t}}^{*}=q_{s_{t}}^{0 *} /\left(1+r_{s_{t}}\right), \tilde{p}_{s_{t}}^{*}=\tilde{q}_{s_{t}}^{*} p_{s_{t}}^{*}, \tilde{b}_{s_{t}}^{*}=\tilde{q}_{s_{t}}^{*} b_{s_{t}}^{*}, \tilde{v}_{s_{t}}^{*}=\tilde{q}_{s_{t}}^{*} v_{s_{t}}^{*}$, and $\tilde{w}^{*}=\sum_{s_{t} \in S} \tilde{v}_{s_{t}}^{*}$.

It holds by definition of the various correspondences involved that

1. for every $h \in H,\left(x^{* h}, a^{* h}, b^{* h}\right) \in \delta^{h}\left(\rho^{*}, q^{*}, v^{* h}\right)$,

2. for every $s_{t} \in S, \sum_{s_{t+1} \in s_{t}^{+}} q_{s_{t+1}}^{*}=1 /\left(1+r_{s_{t}}\right)$,

3. for every $h \in H, s_{t} \in S, v_{s_{t}}^{* h}=\theta^{h} r_{s_{t}} \sum_{h^{\prime} \in H} b_{s_{t}}^{* h^{\prime}}$,

4. for every $s_{T} \in S_{T}, \rho_{\ell\left(s_{T}\right) s_{T}}^{*}=\alpha_{s_{T}}$.

By summing the equalities in 3. over all households, we obtain

$$
\text { for every } s_{t} \in S, \sum_{h \in H} v_{s_{t}}^{* h}=r_{s_{t}} \sum_{h \in H} b_{s_{t}}^{* h} \text {. }
$$

Notice that $\left(x^{* h}, a^{* h}, b^{* h}\right) \in \delta^{h}\left(\rho^{*}, q^{*}, v^{* h}\right)$ implies $\left(x^{* h}, a^{* h}, b^{* h}\right)$ is $\preceq^{h}$-maximal on $\bar{\gamma}^{h}\left(\rho^{*}, q^{*}, v^{* h}\right)=$ $\hat{\gamma}^{h}\left(p^{*}, q^{*}, r, \underline{z}^{*}, \bar{z}^{*}, v^{* h}\right) \cap\left(X^{h} \times A^{h} \times B^{h}\right)$. As argued before, the construction of $A^{h}$ and $B^{h}$ now implies that

$$
\text { for every } h \in H,\left(x^{* h}, a^{* h}, b^{* h}\right) \text { is } \preceq^{h}-\text { maximal on } \hat{\gamma}^{h}\left(p^{*}, q^{*}, r, \underline{z}^{*}, \bar{z}^{*}, v^{* h}\right) \text {. }
$$

STEP 4. Properties of the fixed point: commodities not in $L^{*}$.

Consider $\ell s_{t} \in(L \times S) \backslash L^{*}$. Suppose $\sum_{h \in H} z_{\ell s_{t}}^{* h}<0$. By definition of $\mu_{\ell s_{t}}$, we have $\rho_{\ell s_{t}}^{*}=0$, 
so $p_{\ell s_{t}}^{*}=0$ if $s_{t} \in N_{\ell}^{\mathrm{a}}$ and $\underline{z}_{\ell s_{t}}^{*}=0$ if $s_{t} \in N_{\ell}^{\mathrm{s}}$. In the latter case, $x_{\ell s_{t}}^{* h} \geq e_{\ell s_{t}}^{h}+\underline{z}_{\ell s_{t}}^{*}=e_{\ell s_{t}}^{h}$ for all $h \in H$ by definition of $\bar{\gamma}^{h}$, so $\sum_{h \in H} z_{\ell s_{t}}^{* h} \geq 0$, leading to a contradiction. In the former case, it holds that $\bar{z}_{\ell s_{t}}^{*}=\bar{c}_{\ell s_{t}}$ since $s_{t} \in N_{\ell}^{\mathrm{a}}$. By A2 and A4.6 it holds that $x_{\ell s_{t}}^{* h}=e_{\ell s_{t}}^{h}+\bar{c}_{\ell s_{t}}$ for all $h \in H$, which implies $\sum_{h \in H} z_{\ell s_{t}}^{* h}>0$, leading to a contradiction. Consequently,

$$
\text { for every } \ell s_{t} \in(L \times S) \backslash L^{*}, \sum_{h \in H} z_{\ell s_{t}}^{* h} \geq 0 \text {. }
$$

Consider $\ell s_{t} \in(L \times S) \backslash L^{*}$. Suppose $\sum_{h \in H} z_{\ell s_{t}}^{* h}>0$. By definition of $\mu_{\ell s_{t}}$, we have $\rho_{\ell s_{t}}^{*}=u$ if $s_{t} \in N_{\ell}^{\mathrm{a}}$ and $\rho_{\ell s_{t}}^{*}=1$ if $s_{t} \in N_{\ell}^{\mathrm{s}}$. In the latter case, $x_{\ell s_{t}}^{* h} \leq e_{\ell s_{t}}^{h}+\bar{z}_{\ell s_{t}}^{*}=e_{\ell s_{t}}^{h}$ for all $h \in H$ by definition of $\bar{\gamma}^{h}$, so $\sum_{h \in H} z_{\ell s_{t}}^{* h} \leq 0$, leading to a contradiction. In the former case, $p_{\ell s_{t}}^{*}=u$. Consequently,

$$
\begin{aligned}
& \text { for every } \ell s_{t} \in L \times S, s_{t} \in N_{\ell}^{\mathrm{s}}, \sum_{h \in H} z_{\ell s_{t}}^{* h}=0 \text {, } \\
& \text { for every } \ell s_{t} \in(L \times S) \backslash L^{*} \text { such that } s_{t} \in N_{\ell}^{\mathrm{a}}, \quad \sum_{h \in H} z_{\ell s_{t}}^{* h} \geq 0 \text {, } \\
& \sum_{h \in H} z_{\ell s_{t}}^{* h}>0 \Rightarrow p_{\ell s_{t}}^{*}=u \text {. }
\end{aligned}
$$

STEP 5. Properties of the fixed point: commodity prices.

Consider $\ell s_{t} \in(L \times S) \backslash L^{*}$ such that $s_{t} \in N_{\ell}^{\mathrm{a}}$. Suppose $p_{\ell s_{t}}^{*}=0$. Then we have $\sum_{h \in H} z_{\ell s_{t}}^{* h}>0$ since $\bar{z}_{\ell s_{t}}^{*}=\bar{c}_{\ell s_{t}}$ by definition of $\bar{z}$ and $x_{\ell s_{t}}^{* h}=e_{\ell s_{t}}^{h}+\bar{c}_{\ell s_{t}}$ by A2 and A4.6. By (6.4) it holds that $p_{\ell s_{t}}^{*}=u$, leading to a contradiction. Consequently,

for every $\ell s_{t} \in(L \times S) \backslash L^{*}$ such that $s_{t} \in N_{\ell}^{\mathrm{a}}, p_{\ell s_{t}}^{*}>0$.

Now it holds that

$$
p^{*} \gg 0
$$

since the price of a commodity $\ell s_{T} \in L^{*}$ is equal to $\alpha_{s_{T}}>0$ and the price of a commodity $\ell s_{t^{\prime}}$ with a sticky price is set in previous date-event $s_{t}\left(s_{t^{\prime}}\right)$, so $p_{\ell s_{t^{\prime}}}^{*}=p_{\ell s_{t}\left(s_{t^{\prime}}\right)}^{*}>0$. This argument makes use of the fact that $p_{s_{-1}} \gg 0$ by A6 in case $t=-1$.

STEP 6. Properties of the fixed point: assets.

We now prove by induction that

$$
\text { for every } s_{t} \in S^{+}, \quad \sum_{h \in H} a_{s_{t}}^{* h} \leq 0 \text {. }
$$


By definition of $\bar{\gamma}^{h}$ we have $q_{s_{0}^{+}}^{*} a_{s_{0}^{+}}^{* h} \leq 0$. Let $1_{s_{1}}^{s^{+}}$denote the $s_{1}$-th unit vector in $\mathbb{R}^{s_{0}^{+}}$. It holds that

$$
0 \geq q_{s_{0}^{+}}^{*} \sum_{h \in H} a_{s_{0}^{+}}^{* h} \geq \frac{1}{1+r_{s_{0}}} 1_{s_{1}}^{s_{0}^{+}} \sum_{h \in H} a_{s_{0}^{+}}^{* h}=\frac{1}{1+r_{s_{0}}} \sum_{h \in H} a_{s_{1}}^{* h},
$$

where the second inequality follows by definition of $\nu_{s_{0}}$. We have shown that for all $s_{1} \in s_{0}^{+}$, $\sum_{h \in H} a_{s_{1}}^{* h} \leq 0$, or equivalently $\sum_{h \in H} a_{s_{0}^{+}}^{* h} \leq 0$.

We show next that if, for some $t \in\{1, \ldots, T\}$, for some $s_{t} \in S_{t}, \sum_{h \in H} a_{s_{t}}^{* h} \leq 0$, then for all $s_{t+1} \in s_{t}^{+}, \sum_{h \in H} a_{s_{t+1}}^{* h} \leq 0$, or equivalently $\sum_{h \in H} a_{s_{t}^{+}}^{* h} \leq 0$. By definition of $\bar{\gamma}^{h}$ we have

$$
\sum_{h \in H} q_{s_{t}^{+}}^{*} a_{s_{t}^{+}}^{* h}=\sum_{h \in H}\left(a_{s_{t}}^{* h}-p_{s_{t}^{-}}^{*} z_{s_{t}^{-}}^{* h}-r_{s_{t}^{-}} b_{s_{t}^{-}}^{* h}+v_{s_{t}^{-}}^{* h}\right) \leq 0
$$

where we use the induction hypothesis, the fact that $p_{s_{t}^{-}}^{*} \sum_{h \in H} z_{s_{t}^{-}}^{* h} \geq 0$ by (6.3) and (6.4), and (6.1). By definition of $\nu_{s_{t}}$ it holds that

$$
0 \geq q_{s_{t}^{+}}^{*} \sum_{h \in H} a_{s_{t}^{+}}^{* h} \geq \frac{1}{1+r_{s_{t}}} 1_{s_{t+1}}^{s_{t}^{+}} \sum_{h \in H} a_{s_{t}^{+}}^{* h}=\frac{1}{1+r_{s_{t}}} \sum_{h \in H} a_{s_{t+1}}^{* h}, \quad s_{t+1} \in s_{t}^{+},
$$

so $\sum_{h \in H} a_{s_{t}^{+}}^{* h} \leq 0$. This completes the induction step.

STEP 7. Properties of the fixed point: commodities in $L^{*}$.

Consider $s_{T} \in S_{T}$. It holds that

$$
p_{s_{T}}^{*} \sum_{h \in H} z_{s_{T}}^{* h}=\sum_{h \in H} a_{s_{T}^{+}}^{* h}-\sum_{h \in H}\left(r_{s_{T}} b_{s_{T}}^{* h}-v_{s_{T}}^{* h}\right)=\sum_{h \in H} a_{s_{T}^{+}}^{* h} \leq 0,
$$

where the first equality follows from the definition of $\bar{\gamma}^{h}$ and the second equality from (6.1). By (6.3), (6.4), and (6.5) we have the following result,

$$
\text { for every } s_{T} \in S_{T}, \sum_{h \in H} z_{\ell\left(s_{T}\right) s_{T}}^{* h} \leq 0 .
$$

STEP 8. Properties of the fixed point: asset prices.

For $s_{T+1} \in S_{T+1}$ we have $q_{s_{T+1}}^{*}=1 /\left(1+r_{s_{T+1}}\right)>0$. Suppose $q_{s_{t}}^{*}=0$ for some $s_{t} \in S \backslash\left\{s_{0}\right\}$. Let $s_{T}$ be a period $T$ successor of $s_{t}$. Then $\tilde{p}_{s_{T}}^{*}=\tilde{q}_{s_{T}}^{*} p_{s_{T}}^{*}=0$, so by the by now familiar argument, $x_{\ell\left(s_{T}\right) s_{T}}^{* h}=e_{\ell\left(s_{T}\right) s_{T}}^{h}+\bar{c}_{\ell\left(s_{T}\right) s_{T}}$ for all $h \in H$, leading to a contradiction to (6.6). We have shown that

$$
q^{*} \gg 0
$$


STEP 9. Properties of the fixed point: optimality of choices.

Consider $\ell \in L$ and $s_{T} \in N_{\ell}^{\mathrm{a}}$. For all $h \in H$ we have that

$$
\begin{aligned}
e_{\ell s_{T}}^{h}+\bar{z}_{\ell s_{T}}^{*} & =e_{\ell s_{T}}^{h}+\bar{c}_{\ell s_{T}} \\
& \geq e_{\ell s_{T}}^{h}+\sum_{h^{\prime} \in H}\left(e_{\ell s_{T}}^{h^{\prime}}-\underline{x}_{\ell s_{T}}^{h^{\prime}}\right) \\
& \geq e_{\ell s_{T}}^{h}+\sum_{h^{\prime} \in H}\left(x_{\ell s_{T}}^{* h^{\prime}}-\underline{x}_{\ell s_{T}}^{h^{\prime}}\right) \\
& =x_{\ell s_{T}}^{* h}+\sum_{h^{\prime} \in H \backslash\{h\}}\left(x_{\ell s_{T}}^{* h^{\prime}}-\underline{x}_{\ell s_{T}}^{h^{\prime}}\right)+\left(e_{\ell s_{T}}^{h}-\underline{x}_{\ell s_{T}}^{h}\right) \\
& >x_{\ell s_{T}}^{* h},
\end{aligned}
$$

where the first equality uses $s_{T} \in N_{\ell}^{\mathrm{a}}$, the first inequality uses the definition of $\bar{c}_{\ell_{s_{T}}}$, the second inequality uses (6.6), and the final inequality uses A1 and the definition of $\underline{x}^{h}$.

Suppose, for some $h \in H, \tilde{p}^{*} x^{* h}+r^{*} \tilde{b}^{* h}<\tilde{p}^{*} e^{h}+\tilde{w}^{* h}$. Consider a sequence $\left(x_{n}^{h}\right)_{n \in \mathbb{N}}$ of points in $\bar{X}^{h}$ such that $x_{n}^{h}$ converges to $x^{* h}, x_{\ell s_{T}, n}^{h}>x_{\ell_{s_{T}}}^{* h}$ if $s_{T} \in N_{\ell}^{\mathrm{a}}$, and $x_{\ell s_{t}, n}^{h}=x_{\ell s_{t}}^{* h}$ otherwise. Inequality (6.8) guarantees that $x_{n}^{h}-e^{h} \leq \bar{z}^{*}$ for $n$ sufficiently large. Since $\beta^{h}$ is lower hemi-continuous, there exists a sequence $\left(b_{n}^{h}\right)_{n \in \mathbb{N}}$ of points in $\beta^{h}\left(p^{*}, x_{n}^{h}\right)$ such that $b_{n}^{h} \rightarrow b^{* h}$. For $n$ sufficiently large it holds that $\tilde{p}^{*} x_{n}^{h}+r^{*} \tilde{b}_{n}^{h}<\tilde{p}^{*} e^{h}+\tilde{w}^{* h}$, and one can therefore choose $a_{n}^{h} \in \mathbb{R}^{S^{+}}$such that $\left(x_{n}^{h}, a_{n}^{h}, b_{n}^{h}\right) \in \hat{\gamma}^{h}\left(p^{*}, q^{*}, r, \underline{z}^{*}, \bar{z}^{*}, v^{* h}\right)$. Since $\preceq^{h}$ is monotonic, this contradicts that $\left(x^{* h}, a^{* h}, b^{* h}\right)$ is $\preceq^{h}$-maximal on $\hat{\gamma}^{h}\left(p^{*}, q^{*}, r, \underline{z}^{*}, \bar{z}^{*}, v^{* h}\right)$. Consequently,

for every $h \in H, \tilde{p}^{*} x^{* h}+r^{*} \tilde{b}^{* h}=\tilde{p}^{*} e^{h}+\tilde{w}^{* h}$,

and using (6.2) it follows that

for every $h \in H,\left(x^{* h}, a^{* h}, b^{* h}\right)$ is $\preceq^{h}$-maximal on $\gamma^{h}\left(p^{*}, q^{*}, r, \underline{z}^{*}, \bar{z}^{*}, v^{* h}\right)$, so (a) of Definition 5.1 holds.

STEP 10. Lifting the upper bound on prices.

Let $\left(u_{n}\right)_{n \in \mathbb{N}}$ be a sequence of points such that $u_{n} \geq \max \left\{1, \max _{s_{T} \in S_{T}} \alpha_{s_{T}}, \max _{\ell \in L} p_{\ell s_{-1}}\right\}$ and $u_{n} \rightarrow \infty$. Let $\bar{R}_{n}, A_{n}, B_{n}$, and $V_{n}$ denote the corresponding compactified sets of endogenous variables as constructed in Step 1 and let $\left(\varphi_{n}\right)_{n \in \mathbb{N}}$ be a sequence of fixed point correspondences as constructed in Step 2. Let $\left(\rho_{n}^{*}, q_{n}^{*}, v_{n}^{*}, x_{n}^{*}, a_{n}^{*}, b_{n}^{*}\right)_{n \in \mathbb{N}}$ be a corresponding sequence of fixed points, whose existence is shown in Step 3. For $n \in \mathbb{N}$, we define $p_{n}^{*}=$ $p\left(\rho_{n}^{*}\right), \underline{z}_{n}^{*}=\underline{z}\left(\rho_{n}^{*}\right), \bar{z}_{n}^{*}=\bar{z}\left(\rho_{n}^{*}\right)$, and $z_{n}^{*}=x_{n}^{*}-e$. Moreover, we define the present-value variables $\tilde{q}_{n}^{*}, \tilde{p}_{n}^{*}, \tilde{b}_{n}^{*}, \tilde{v}_{n}^{*}$, and $\tilde{w}_{n}^{*}$ by setting, for $s_{t} \in S, \tilde{q}_{s_{t}, n}^{*}=q_{s_{t}, n}^{0 *} /\left(1+r_{s_{t}}\right), \tilde{p}_{s_{t}, n}^{*}=\tilde{q}_{s_{t}, n}^{*} p_{s_{t}, n}^{*}$, $\tilde{b}_{s_{t}, n}^{*}=\tilde{q}_{s_{t}, n}^{*} b_{s_{t}, n}^{*}, \tilde{v}_{s_{t}, n}^{*}=\tilde{q}_{s_{t}, n}^{*} v_{s_{t}, n}^{*}$, and $\tilde{w}_{n}^{*}=\sum_{s_{t} \in S} \tilde{v}_{s_{t}, n}^{*}$ 
Suppose for every $n \in \mathbb{N}$ there is $\ell s_{t} \in L \times S$ such that $\sum_{h \in H} z_{\ell s_{t}, n}^{* h}>0$. We will derive a contradiction in Steps 11-13.

SteP 11. Construction of a convergent subsequence.

For every $n \in \mathbb{N}$, for every $h \in H$, let $\underline{b}_{n}^{h}$ be a minimal element of $\beta^{h}\left(p_{n}^{*}, x_{n}^{* h}\right)$, so there is no $b^{h} \in \beta^{h}\left(p_{n}^{*}, x_{n}^{* h}\right)$ with $b^{h}<\underline{b}_{n}^{h}$. Since $\beta^{h}$ is closed by A4.1, such a $\underline{b}_{n}^{h}$ exists. We define $\underline{\tilde{b}}_{n}^{h}$ by setting $\tilde{\underline{b}}_{s_{t}, n}^{h}=\tilde{q}_{s_{t}, n}^{*} \underline{b}_{s_{t}, n}^{h}$ for $s_{t} \in S$. We have

for every $n \in \mathbb{N}$, for every $h \in H, r \underline{\tilde{b}}_{n}^{h}=r \tilde{b}_{n}^{* h}$,

since it clearly holds that $r \underline{b}_{n}^{h} \leq r \tilde{b}_{n}^{* h}$, whereas a strict inequality would lead to a contradiction as in the proof of inequality (6.7).

We divide $\tilde{p}_{n}^{*}, \underline{\tilde{b}}_{n}, \tilde{v}_{n}^{*}$, and $\tilde{w}_{n}^{*}$ by $\left\|\tilde{p}_{n}^{*}\right\|_{\infty}$ and denote the resulting variables by $\hat{p}_{n}, \hat{b}_{n}$, $\hat{v}_{n}$, and $\hat{w}_{n}$. We claim that these variables are bounded. It is obvious that the sequence $\left(\hat{p}_{n}\right)_{n \in \mathbb{N}}$ is bounded.

We show next that the sequence $\left(\hat{b}_{n}\right)_{n \in \mathbb{N}}$ is bounded and start by showing that this sequence is bounded from below. We have that $\underline{b}_{n}^{h} \in \beta^{h}\left(p_{n}^{*}, x_{n}^{* h}\right)$ and $\underline{f}^{h}\left(p_{n}^{*}, x_{n}^{* h}\right) \leq \underline{b}_{n}^{h}$. By the homogeneity as stated in A4.3, it holds that $\underline{\hat{b}}_{n}^{h} \in \beta^{h}\left(\hat{p}_{n}, x_{n}^{* h}\right)$. By A4.2 it holds that $\underline{f}^{h}\left(\hat{p}_{n}, x_{n}^{* h}\right) \leq \underline{\hat{b}}_{n}^{h}$. We define $P=\left\{p \in \mathbb{R}_{+}^{L S} \mid\|p\|_{\infty}=1\right\}$. Since the set $P \times \bar{X}^{h}$ is compact and $\underline{f}^{h}$ is continuous, the set $\underline{f}^{h}\left(P \times \bar{X}^{h}\right)$ is compact, so bounded from below. It follows that the sequence $\left(\underline{b}_{n}\right)_{n \in \mathbb{N}}$ is bounded from below. To show that this sequence is bounded from above, we show first that $\underline{\underline{b}}_{n}^{h} \leq \bar{f}^{h}\left(\hat{p}_{n}, x_{n}^{* h}\right)$. Suppose not, then by A4.2 we have $\hat{b}_{n}^{h}=\min \left\{\bar{f}^{h}\left(\hat{p}_{n}, x_{n}^{* h}\right), \underline{\hat{b}}_{n}^{h}\right\} \in \beta^{h}\left(\hat{p}_{n}, x_{n}^{* h}\right)$ and $\hat{b}_{n}^{h}<\underline{\hat{b}}_{n}^{h}$. We define $b_{n}^{h} \in \mathbb{R}^{S}$ by setting

$$
b_{s_{t}, n}^{h}=\frac{\left\|\tilde{p}_{n}^{*}\right\|_{\infty}}{\tilde{q}_{s_{t}, n}^{*}} \hat{b}_{s_{t}, n}^{h}, \quad s_{t} \in S .
$$

Notice that $\tilde{q}_{s_{t}, n}^{*}>0$ by (6.7), so $b_{n}^{h}$ is well-defined. Then it holds by A4.3 that $b_{n}^{h} \in$ $\beta^{h}\left(p_{n}^{*}, x_{n}^{* h}\right)$, whereas $b_{n}^{h}<\underline{b}_{n}^{h}$, a contradiction to the choice of $\underline{b}_{n}^{h}$. Consequently, it holds that $\underline{\hat{b}}_{n}^{h} \leq \bar{f}^{h}\left(\hat{p}_{n}, x_{n}^{* h}\right)$. Since the set $P \times \bar{X}^{h}$ is compact and $\bar{f}^{h}$ is continuous, the set $\bar{f}^{h}\left(P \times X^{h}\right)$ is compact, so bounded from above. It follows that the sequence $\left(\underline{b}_{n}\right)_{n \in \mathbb{N}}$ is bounded from above.

Since, by (6.10), for $s_{t} \in S, \hat{v}_{s_{t}, n}^{h}=\theta^{h} r_{s_{t}} \sum_{h^{\prime} \in H} \hat{b}_{s_{t}, n}^{h^{\prime}}$, and $\left(\hat{b}_{n}\right)_{n \in \mathbb{N}}$ is bounded, it follows that the sequence $\left(\hat{v}_{n}\right)_{n \in \mathbb{N}}$ is bounded. Next, since $\hat{w}^{h}=\sum_{s_{t} \in S} \hat{v}_{s t}^{h}$, it follows that the sequence $\left(\hat{w}_{n}\right)_{n \in \mathbb{N}}$ is bounded.

The boundedness of the various sequences of endogenous variables implies that, without loss of generality, $\left(\hat{p}_{n}, \tilde{q}_{n}^{*}, \underline{z}_{n}^{*}, \bar{z}_{n}^{*}, \hat{w}_{n}, x_{n}^{*}, \underline{\hat{b}}_{n}\right)$ is a convergent sequence with limit, say, $\left(\hat{p}, \tilde{q}^{*}, \underline{z}^{*}, \bar{z}^{*}, \hat{w}, x^{*}, \underline{\hat{b}}\right)$. Moreover, without loss of generality, there is $\ell s_{t} \in L \times S$ such that for all $n \in \mathbb{N}, z_{\ell s_{t}, n}^{* h}>0$. By (6.4) we have that $s_{t} \in N_{\ell}^{\mathrm{a}}$ and $p_{\ell s_{t}, n}^{*}=u_{n}$. 
STEP 12. Continuity of demand at the limit.

We argue next that $\hat{p} \underline{z}^{*}<0$. We achieve this by showing that there is a commodity $\ell s_{t}$ such that $s_{t} \in N_{\ell}^{\mathrm{a}}$ and $\hat{p}_{\ell s_{t}}>0$.

Consider $\ell s_{t^{\prime}} \in L \times S$ such that $\hat{p}_{\ell s_{t^{\prime}}}=1$. If $s_{t^{\prime}} \in N_{\ell}^{\mathrm{a}}$, then we are done. If $s_{t^{\prime}} \in N_{\ell}^{\mathrm{s}}$, then let $s_{t}$ be the date-event where the price of $\ell s_{t^{\prime}}$ is set. If $t \geq 0$, then $s_{t} \in N_{\ell}^{\mathrm{a}}$ and

$$
\hat{p}_{\ell s_{t}}=\lim _{n \rightarrow \infty} \hat{p}_{\ell s_{t}, n} \geq \lim _{n \rightarrow \infty} \hat{p}_{\ell s_{t^{\prime}}, n}=\hat{p}_{\ell s_{t^{\prime}}}=1,
$$

where the inequality uses the fact that $p_{\ell s_{t}, n}^{*}=p_{\ell s_{t^{\prime}}, n}^{*}$ and $\tilde{q}_{\ell s_{t}, n}^{*} \geq \tilde{q}_{\ell s_{t^{\prime}}, n}^{*}$. It follows that $\hat{p}_{\ell s_{t}}=1$. If $t=-1$, then

$$
\hat{p}_{\ell s_{t^{\prime}}}=\lim _{n \rightarrow \infty} \frac{\tilde{p}_{\ell s_{t^{\prime}}, n}^{*}}{\left\|\tilde{p}_{n}^{*}\right\|_{\infty}}=\lim _{n \rightarrow \infty} \frac{\tilde{q}_{s_{t^{\prime}}, n}^{*} p_{\ell s_{t^{\prime}}, n}^{*}}{\left\|\tilde{p}_{n}^{*}\right\|_{\infty}}=\lim _{n \rightarrow \infty} \frac{\tilde{q}_{s_{t^{\prime}}, n}^{*} p_{\ell s_{-1}}}{\left\|\tilde{p}_{n}^{*}\right\|_{\infty}}=1,
$$

so $\lim _{n \rightarrow \infty}\left\|\tilde{p}_{n}^{*}\right\|_{\infty}=\tilde{q}_{s_{t^{\prime}}}^{*} p_{\ell s_{-1}}$. Let $s_{T}$ be a date-event such that $\tilde{q}_{s_{T}}^{*}>0$. Then, for every $n \in \mathbb{N}$,

$$
\left\|\tilde{p}_{n}^{*}\right\|_{\infty} \geq \tilde{q}_{s_{T}, n}^{*} p_{\ell\left(s_{T}\right) s_{T}}^{*}=\tilde{q}_{s_{T}, n}^{*} \alpha_{s_{T}}
$$

so $\tilde{q}_{s^{\prime}}^{*} p_{\ell_{-1}}=\lim _{n \rightarrow \infty}\left\|\tilde{p}_{n}^{*}\right\|_{\infty} \geq \tilde{q}_{s_{T}}^{*} \alpha_{s_{T}}>0$ and

$$
\hat{p}_{\ell\left(s_{T}\right) s_{T}}=\frac{\tilde{q}_{s_{T}}^{*} \alpha_{s_{T}}}{\tilde{q}_{s_{t^{\prime}}}^{*} p_{\ell s_{-1}}}>0 .
$$

Since $s_{T} \in N_{\ell\left(s_{T}\right)}^{\mathrm{a}}$, we have shown that $\hat{p} \underline{z}^{*}<0$.

Let $\Xi^{h}$ be the set of all points in the sequence $\left(\hat{p}_{n}, r, \underline{z}_{n}^{*}, \bar{z}_{n}^{*}, \hat{w}_{n}^{h}\right)_{n \in \mathbb{N}}$ and its limit $\left(\hat{p}, r, \underline{z}^{*}, \bar{z}^{*}, \hat{w}^{h}\right)$. Let $\underline{B}^{h}$ be a compact set containing all the points in the sequence $\left(\underline{\hat{b}}_{n}^{h}\right)_{n \in \mathbb{N}}$ and its limit $\left(\underline{\hat{b}}^{h}\right)$ in its interior. We define $\underline{\gamma}^{h}: \Xi^{h} \rightarrow \bar{X}^{h} \times \underline{B}^{h}$ by $\underline{\gamma}^{h}\left(\xi^{h}\right)=\tilde{\gamma}^{h}\left(\xi^{h}\right) \cap\left(\bar{X}^{h} \cap \underline{B}^{h}\right)$. Since $\tilde{\gamma}^{h}$ is lower hemi-continuous on $\Xi^{h}$ by Lemma 4.1 and for all $\xi^{h} \in \Xi^{h}, \operatorname{int}\left(\tilde{\gamma}^{h}\left(\xi^{h}\right)\right) \cap \operatorname{int}\left(\bar{X}^{h} \cap \underline{B}^{h}\right) \neq \emptyset$, it follows from Hildenbrand (1974), Problem 6, p. 35, that $\underline{\gamma}^{h}$ is lower hemi-continuous. Since $\bar{X}^{h} \times \underline{B}^{h}$ is compact and $\tilde{\gamma}^{h}$ is closed by Lemma 4.1, we have that $\underline{\gamma}^{h}$ has a closed graph, so is upper hemi-continuous. We define $\underline{\delta}^{h}: \Xi^{h} \rightarrow \bar{X}^{h} \times \underline{B}^{h}$ by $\operatorname{defining} \underline{\delta}^{h}\left(\xi^{h}\right)$ as the set of $\preceq^{h}$-maximal elements on $\underline{\gamma}^{h}\left(\xi^{h}\right)$. An application of the maximum theorem demonstrates that $\underline{\delta}^{h}$ is an upper hemi-continuous correspondence.

STEP 13. Deriving a contradiction.

Since for $n \in \mathbb{N},\left(x_{n}^{* h}, \hat{b}_{n}^{h}\right) \in \underline{\delta}^{h}\left(\hat{p}_{n}, r, \underline{z}_{n}^{*}, \bar{z}_{n}^{*}, \hat{w}_{n}^{h}\right)_{n \in \mathbb{N}}$, it follows by upper hemi-continuity of $\underline{\delta}^{h}$ that $\left(x^{* h}, \hat{b}^{h}\right) \in \underline{\delta}^{h}\left(\hat{p}, r, \underline{z}^{*}, \bar{z}^{*}, \hat{w}^{h}\right)$.

Consider $\ell s_{t} \in L \times S$ such that for all $n \in \mathbb{N}, p_{\ell s_{t}, n}^{*}=u_{n}$ and let $s_{T}$ be a successor of $s_{t}$ in period $T$ or equal to $s_{t}$ in case $t=T$. We show that $\hat{p}_{\ell\left(s_{T}\right) s_{T}}=0$. First, consider 
the case where $\tilde{q}_{s_{t}}^{*}=0$. Then it holds that $\tilde{q}_{s_{T}}^{*}=0$ and $p_{\ell\left(s_{T}\right) s_{T}}=\alpha_{s_{T}}$. Moreover, $\left\|\tilde{p}_{n}^{*}\right\|_{\infty}$ is bounded away from zero, since $\left\|\tilde{p}_{n}^{*}\right\|_{\infty} \geq \tilde{q}_{\bar{s}_{T}, n}^{*} \alpha_{\bar{s}_{T}} \rightarrow \tilde{q}_{\bar{s}_{T}}^{*} \alpha_{\bar{s}_{T}}>0$, where $\bar{s}_{T}$ is chosen such that $\tilde{q}_{\bar{s}_{T}}^{*}>0$. We therefore have that

$$
\hat{p}_{\ell\left(s_{T}\right) s_{T}}=\lim _{n \rightarrow \infty} \hat{p}_{\ell\left(s_{T}\right) s_{T}, n}=\lim _{n \rightarrow \infty} \frac{\tilde{q}_{s_{T}, n}^{*} p_{\ell\left(s_{T}\right) s_{T}, n}^{*}}{\left\|\tilde{p}_{n}^{*}\right\|_{\infty}}=0 .
$$

Second, consider the case where $\tilde{q}_{s_{t}}^{*}>0$. Since $p_{\ell s_{t}, n}^{*}=u_{n}$, it follows that $\tilde{p}_{\ell s_{t}, n}^{*}=$ $\tilde{q}_{s_{t}, n}^{*} p_{\ell s_{t}, n}^{*} \rightarrow \infty$ as $n \rightarrow \infty$, so $\left\|\tilde{p}_{n}^{*}\right\|_{\infty} \rightarrow \infty$ as $n \rightarrow \infty$. We therefore have that

$$
\hat{p}_{\ell\left(s_{T}\right) s_{T}}=\lim _{n \rightarrow \infty} \hat{p}_{\ell\left(s_{T}\right) s_{T}, n}=\lim _{n \rightarrow \infty} \frac{\tilde{q}_{s_{T}, n}^{*} p_{\ell\left(s_{T}\right) s_{T}, n}^{*}}{\left\|\tilde{p}_{n}^{*}\right\|_{\infty}}=\frac{\tilde{q}_{s_{T}}^{*} \alpha_{s_{T}}}{\lim _{n \rightarrow \infty}\left\|\tilde{p}_{n}^{*}\right\|_{\infty}}=0 .
$$

Consider $\left(x^{h}, b^{h}\right) \in \underline{\delta}^{h}\left(\hat{p}, r, \underline{z}^{*}, \bar{z}^{*}, \hat{w}^{h}\right)$. Since $\hat{p}_{\ell\left(s_{T}\right) s_{T}}=0$, we have that

$$
x_{\ell\left(s_{T}\right) s_{T}}^{h}=e_{\ell\left(s_{T}\right) s_{T}}^{h}+\bar{z}_{\ell\left(s_{T}\right) s_{T}}^{*}=e_{\ell\left(s_{T}\right) s_{T}}^{h}+c_{\ell\left(s_{T}\right) s_{T}} .
$$

Since $\underline{\delta}^{h}$ is upper hemi-continuous at $\left(\hat{p}, r, \underline{z}^{*}, \bar{z}^{*}, \hat{w}^{h}\right)$, we have that

$$
\sum_{h \in H} z_{\ell\left(s_{T}\right) s_{T}, n}^{* h} \rightarrow \sum_{h \in H} c_{\ell\left(s_{T}\right) s_{T}}>0,
$$

a contradiction to (6.6).

STEP 14. Market clearing for commodities.

We have shown that there is $n \in \mathbb{N}$ such that for every $\ell s_{t} \in L \times S, \sum_{h \in H} z_{\ell s_{t}, n}^{* h} \leq 0$. We fix such an $n$, and omit it from the notation. Suppose $\sum_{h \in H} z^{* h}<0$. It holds that

$$
\tilde{p}^{*} \sum_{h \in H} z^{* h}<0
$$

where the inequality uses (6.5) and (6.7). At the same time, we have by (6.9) and (6.1) that

$$
\begin{aligned}
\tilde{p}^{*} \sum_{h \in H} z^{* h} & =r^{*} \sum_{h \in H} \tilde{b}^{* h}-\tilde{w}^{* h} \\
& =\sum_{s_{t} \in S} \tilde{q}_{s t}^{*}\left(r_{s_{t}} \sum_{h \in H} b^{* h}-\sum_{h \in H} v^{* h}\right)=0 .
\end{aligned}
$$

We have derived a contradiction. Consequently, it holds that $\sum_{h \in H} x^{* h}=\sum_{h \in H} e^{h}$ and we have shown (b) of Definition 5.1.

STEP 15. Market clearing for assets.

We use induction to show (c) of Definition 5.1. For every $s_{T+1} \in S_{T+1}$, by definition of $\gamma^{h}$, $h \in H$,

$$
\sum_{h \in H} a_{s_{T+1}}^{* h}=p_{s_{T+1}^{-}}^{*} \sum_{h \in H}\left(x_{s_{T+1}^{-}}^{* h}-e_{s_{T+1}^{-}}^{h}\right)+r_{s_{T+1}^{-}} \sum_{h \in H} b_{s_{T+1}^{-}}^{* h}-\sum_{h \in H} v_{s_{T+1}^{-}}^{* h}=0,
$$


where the last equality uses (b) of Definition 5.1 and (6.1).

Assume, for some $t \in\{0, \ldots, T\}$, we have shown that for every $s_{t+1} \in S_{t+1}, \sum_{h \in H} a_{s_{t+1}}^{* h}=$ 0 . For every $s_{t} \in S_{t}$, by definition of $\gamma^{h}, h \in H$,

$$
\sum_{h \in H} a_{s_{t}}^{* h}=p_{s_{t}^{-}}^{*} \sum_{h \in H}\left(x_{s_{t}^{-}}^{* h}-e_{s_{t}^{-}}^{h}\right)+r_{s_{t}^{-}} \sum_{h \in H} b_{s_{t}^{-}}^{* h}-\sum_{h \in H} v_{s_{t}^{-}}^{* h}+\sum_{s_{t+1} \in s_{t}^{+}} q_{s_{t+1}}^{*} \sum_{h \in H} a_{s_{t+1}}^{* h}=0,
$$

where the last equality uses (b) of Definition 5.1, (6.1), and the induction hypothesis. This completes the proof of Theorem 6.1.

By Theorem 5.2 the following corollary follows at once.

Corollary 6.2 For all $s_{T} \in S_{T}$, assume $s_{T} \in \cup_{\ell \in L} N_{\ell}^{\mathrm{a}}$. Let $L^{*}$ be a set with one flexible price commodity for each terminal date-event. For each choice of $\alpha \in \mathbb{R}_{++}^{S_{T}}$, there is a sticky price equilibrium $\left(p^{*}, q^{*}, \underline{z}^{*}, \bar{z}^{*}, v^{*}, x^{*}, a^{*}, b^{*}\right)$ satisfying, for all $\ell s_{T} \in L^{*}, p_{\ell_{s_{T}}}^{*}=\alpha_{s_{T}}$.

What is the intuition underlying the results of Theorem 6.1 and Corollary 6.2? Counting equations and unknowns in Definition 5.1 of a parametrized sticky price equilibrium reveals that there are $|L||S|$ commodity market clearing conditions and $|L||S|$ variables $\rho_{\ell_{s_{t}}}$. Moreover, there are $\left|S^{+}\right|$asset market clearing conditions and $\left|S^{+}\right|$asset prices $q_{s_{t}}$. There are $|S|+\left|S_{T}\right|$ budget constraints in (2.1), leading to $|S|+\left|S_{T}\right|$ Walras' laws, inducing $|S|+\left|S_{T}\right|$ degrees of freedom for equilibrium. The policy of the bank involves setting $|S|$ interest rates, leading to $|S|$ no-arbitrage conditions in Definition 5.1, reducing the degrees of freedom for equilibrium by $|S|$, resulting in $\left|S_{T}\right|$ degrees of indeterminacy.

One way to lift the $\left|S_{T}\right|$ degrees of indeterminacy has been been suggested by Magill and Quinzii (2010) in a model without price stickiness. Their suggestion essentially boils down to choosing $\left|S_{T}\right|$ additional instruments, in their case by having the bank not only controlling the short-term interest rates, but in addition the interest rates on bonds with longer maturity. Although it is not completely evident that the needed controllability requirements carry over to the sticky price framework, the general principle that more instruments are needed to lower the degree of indeterminacy holds. But since the number $\left|S_{T}\right|$ should be thought of as huge, the date-event tree should in principle include all date-events on which agents can condition their actions, the extent to which additional instruments help to reduce nominal and real indeterminacy remains an open issue.

\section{Degrees of Nominal and Real Indeterminacy}

Corollary 6.2 shows that for each choice of $\alpha \in \mathbb{R}_{++}^{S_{T}}$ there is a sticky price equilibrium with, for all $\ell s_{T} \in L^{*}, p_{\ell_{s_{T}}}^{*}=\alpha_{s_{T}}$. The equilibrium nominal price level at terminal date-events is 
arbitrary, irrespective of the interest rate policy by the bank.

The case where all prices are flexible has been studied in Drèze and Polemarchakis (2001). The absence of imperfections in price formation and the homogeneity assumptions imposed on the transactions technology imply that the whole analysis there can be done in terms of present-value prices. Each equilibrium in terms of present-value prices leads to an $S_{T}$-dimensional set of equilibria.

To illustrate this fact, consider, for the sake of concreteness, an economy with two periods, and let $\left(p_{s_{0}}^{0},\left(p_{s_{1}}^{0}\right)_{s_{1} \in S_{1}}\right)$ be present-value equilibrium prices. In terms of nominal prices, we have that

$$
\begin{aligned}
& p_{s_{0}}^{0}=p_{s_{0}}, \\
& p_{s_{1}}^{0}=q_{s_{1}}^{0} p_{s_{1}}=q_{s_{1}} p_{s_{1}}, \quad s_{1} \in S_{1} .
\end{aligned}
$$

By the homogeneity assumption on the transactions technology, we find that the presentvalue equilibrium prices $\left(p_{s_{0}}^{0},\left(p_{s_{1}}^{0}\right)_{s_{1} \in S_{1}}\right)$ induce the $\left(S_{1}-1\right)$-dimensional set of nominal equilibrium prices $\left(p_{s_{0}},\left(p_{s_{1}}\right)_{s_{1} \in S_{1}}\right)$ given by

$$
\begin{aligned}
& p_{s_{0}}=p_{s_{0}}^{0}, \\
& p_{s_{1}}=\frac{1}{q_{s_{1}}} p_{s_{1}}^{0}, \quad s_{1} \in S_{1},
\end{aligned}
$$

whenever $q \in \mathbb{R}_{++}^{S_{1}}$ satisfies $\sum_{s_{1} \in S_{1}} q_{s_{1}}=1$, so induces $S_{1}-1$ degrees of freedom.

Whenever $\left(p_{s_{0}},\left(p_{s_{1}}\right)_{s_{1} \in S_{1}}\right)$ are nominal equilibrium prices, so is $\left(\lambda p_{s_{0}},\left(\lambda p_{s_{1}}\right)_{s_{1} \in S_{1}}\right)$ for any $\lambda>0$, which adds one degree of freedom and shows that each equilibrium in terms of present-value prices leads to an $S_{1}$-dimensional set of equilibria. All these equilibria induce the same equilibrium allocation, so there is only nominal indeterminacy and no real indeterminacy of equilibrium in this case.

The same $S_{1}$-dimensional set of equilibria results from Corollary 6.2, though the parametrization chosen there is different. According to Corollary 6.2, one can choose a flexible price commodity $\ell\left(s_{1}\right)$ for each date-event $s_{1} \in S_{1}$, a vector of price levels $\alpha \in \mathbb{R}_{++}^{S_{1}}$ and have an equilibrium with nominal prices given by $p_{\ell\left(s_{1}\right)}=\alpha_{s_{1}}$.

Although the bank cannot control the price level by interest rate policy, it can control expected inflation. To illustrate this, suppose the economy is stationary, meaning that present-value equilibrium prices are constant up to a discount factor $\delta$,

$$
p_{s_{1}}^{0}=\pi_{s_{1}} \delta p_{s_{0}}^{0}, \quad s_{1} \in S_{1}
$$

where $\pi_{s_{1}}$ is the probability of occurrence of state $s_{1}$. We have that the harmonic mean of period one prices is equal to

$$
H\left(p_{S_{1}}\right)=\frac{1}{\sum_{s_{1} \in S_{1}} \frac{\pi_{s_{1}}}{p_{s_{1}}}}=\frac{1}{\sum_{s_{1} \in S_{1}} \frac{\pi_{s_{1}} q_{s_{1}}}{p_{s_{1}}^{0}}}=\frac{1}{\sum_{s_{1} \in S_{1}} \frac{q_{s_{1}}}{\delta p_{s_{0}}}}=\frac{1}{\frac{1}{\delta p_{s_{0}}\left(1+r_{s_{0}}\right)}}=\delta p_{s_{0}}\left(1+r_{s_{0}}\right) .
$$


Expected inflation is equal to $\delta\left(1+r_{s_{0}}\right)$. Higher nominal interest rates lead to higher expected inflation. The variance of inflation, however, can be arbitrarily high and is not controlled by the interest rate. Whenever there are two or more date-events in period one, arbitrarily high inflation rates are caused by values of $q_{s_{1}}$ arbitrarily close to zero. Since expected inflation is controlled, high inflation in some date-events has to be compensated by low inflation or even deflation in other date-events, as it holds that $\sum_{s_{1} \in S} q_{s_{1}}=1 /\left(1+r_{s_{0}}\right)$.

This reasoning extends to the general model with $T+1$ periods.

These conclusions change when the set of sticky-price commodities is non-empty. By Corollary 6.2 there is still an $S_{T}$-dimensional set of equilibria. However, the route to demonstrate this result via proving the existence of an equilibrium in terms of present-value prices and next generating an $S_{T}$-dimensional set of nominal equilibrium prices by appropriate choices of $q$ and $\lambda$ is blocked. Multiplications of all prices by $\lambda$ violates price stickiness if $s_{0} \in \cup_{\ell \in L} N_{\ell}^{\mathrm{s}}$, whereas it can easily happen that no choice for $q$ is consistent with price stickiness. The existence proof of Theorem 6.1 is therefore by means of correspondences that are formulated in terms of nominal prices.

To illustrate the implications of Theorem 6.1 and Corollary 6.2, consider again an economy with two periods, one commodity per date-event, but now a sticky price in period 0 , and flexible prices in period 1. By Corollary 6.2 one can choose an arbitrary vector of prices $\alpha \in \mathbb{R}_{++}^{S_{1}}$ and have an equilibrium with nominal prices given by $p_{s_{1}}=\alpha_{s_{1}}$ for $s_{1} \in S_{1}$. Since the period 0 price is sticky, this result implies that it is not even possible to control expected inflation by nominal interest rate policy. Expected inflation can be arbitrarily high or low, irrespective of nominal interest rates, as the vector $\alpha$ can be arbitrarily chosen.

How are markets equilibrated in such an economy? The equilibrium state prices $q_{s_{1}}$ should satisfy $\sum_{s_{1} \in S_{1}} q_{s_{1}}=1 /\left(1+r_{s_{0}}\right)$ and, since all prices in period 1 are flexible, should be such that at equilibrium present-value prices $p_{s_{1}}^{0}=q_{s_{1}} p_{s_{1}}$ there is zero aggregate net trade across future date-events. Relatively high values of $\alpha_{s_{1}}$ go together with low prices $q_{s_{1}}$ and vice versa. When the vector $\alpha$ has high values across the board, with a sticky price in period 0 , the only channel to general zero intertemporal aggregate trade is demand rationing in period 0 . When expected inflation is high and the price in period 0 does not adjust, consumers will face demand rationing in period 0 . Vice versa, with low expected inflation, or even expected deflation, a sticky price in period 0 leads to lack of demand and supply rationing in period 0 .

In the simple case with one commodity per date-event and a sticky price in period 0 , we can generate $S_{1}-1$ degrees of nominal indeterminacy by an appropriate choice of the asset prices $q$ in the following way. Let $\left(p_{s_{0}}^{0},\left(p_{s_{1}}^{0}\right)_{s_{1} \in S_{1}}\right)$ be present-value equilibrium prices. Since the price in period 0 is assumed to be sticky, it holds that $p_{s_{0}}^{0}=p_{s_{0}}=p_{s_{-1}}$. Any 
choice of $q \in \mathbb{R}_{++}^{S_{1}}$ satisfying $\sum_{s_{1} \in S_{1}} q_{s_{1}}=1 /\left(1+r_{s_{0}}\right)$ leads to nominal equilibrium prices

$$
p_{s_{1}}=\frac{1}{q_{s_{1}}} p_{s_{1}}^{0}, \quad s_{1} \in S_{1},
$$

at unchanged rationing schemes and allocation of commodities, thereby generating $S_{1}-1$ degrees of nominal indeterminacy.

Consider a particular choice for asset prices, say $\bar{q} \in \mathbb{R}_{++}^{S_{1}}$ satisfying $\sum_{s_{1} \in S_{1}} \bar{q}_{s_{1}}=$ $1 /\left(1+r_{s_{0}}\right)$, and let $\bar{p}_{S_{1}}$ be the corresponding nominal commodity prices in period 1 . For any $\lambda>0$ it holds by Corollary 6.2 that there is a sticky price equilibrium with $p_{S_{1}}=\lambda \bar{p}_{S_{1}}$. Due to the requirement $\sum_{s_{1} \in S_{1}} \bar{q}_{s_{1}}=1 /\left(1+r_{s_{0}}\right)$, the present-value equilibrium prices corresponding to different values of $\lambda$ are all distinct. Since $p_{s_{0}}$ is sticky, it also holds that price ratios $\left(p_{s_{1}}^{0} / p_{s_{0}}^{0}\right)_{s_{1} \in S_{1}}$ are distinct for distinct values of $\lambda$. There is a one-dimensional set of equilibria exhibiting real indeterminacy as an increase or a decrease of $\lambda$ leads on average to overall increases or decreases in present-value prices for future commodities, thereby affecting the budget set.

The general message, however, is that in the presence of price stickiness there are $S_{T}$ degrees of real indeterminacy, so all indeterminacy is real. To show such a result, we make the assumption that at every date-event there is at least one commodity with a sticky price. The next result demonstrates that sticky price equilibria corresponding to different choices for $\alpha$ have present-value prices which are not proportional to each other.

TheOrem 7.1 For all $s_{t} \in S$, assume $s_{t} \in \cup_{\ell \in L} N_{\ell}^{\mathrm{s}}$, and for all $s_{T} \in S_{T}$, assume $s_{T} \in$ $\cup_{\ell \in L} N_{\ell}^{\mathrm{a}}$. Let $L^{*}$ be a set with one flexible price commodity for each terminal date-event. Let $(p, q, \underline{z}, \bar{z}, v, x, a, b)$ and $\left(\bar{p}, \bar{q}, \underline{z}^{\prime}, \bar{z}^{\prime}, \bar{v}, \bar{x}, \bar{a}, \bar{b}\right)$ be sticky price equilibria such that $p_{L^{*}}=\alpha$ and $\bar{p}_{L^{*}}=\bar{\alpha}$. If $\alpha \neq \bar{\alpha}$, then there is no $\lambda \geq 0$ such that $p^{0}=\lambda \bar{p}^{0}$.

ProOF: Assume $p^{0}=\lambda \bar{p}^{0}$ for some $\lambda \geq 0$. In the sequel we make repeatedly use of the facts shown in the proof of Theorem 6.1 that according to (6.5) $p, \bar{p} \gg 0$ and according to (6.7) $q, \bar{q} \gg 0$.

Let $\ell \in L$ be such that $s_{0} \in N_{\ell}^{\mathrm{s}}$. We have that

$$
p_{\ell s_{0}}^{0}=p_{\ell s_{0}}=p_{\ell s_{-1}}=\bar{p}_{\ell s_{0}}=\bar{p}_{\ell s_{0}}^{0} .
$$

These inequalities show that $\lambda=1$, so $p^{0}=\bar{p}^{0}$, and in particular $p_{s_{0}}=\bar{p}_{s_{0}}$.

Next we prove by induction on $t$ that for all $s_{t} \in S_{t}, p_{s_{t}}=\bar{p}_{s_{t}}$ and $q_{s_{t}}^{0}=\bar{q}_{s_{t}}^{0}$.

Consider some $s_{1} \in S_{1}$ and let $\ell \in L$ be such that $s_{1} \in N_{\ell}^{\mathrm{s}}$. We have that

$$
\begin{aligned}
& p_{\ell s_{1}}^{0}=q_{s_{1}}^{0} p_{\ell s_{1}}=q_{s_{1}}^{0} p_{\ell s_{0}}, \\
& \bar{p}_{\ell s_{1}}^{0}=\bar{q}_{s_{1}}^{0} \bar{p}_{\ell s_{1}}=\bar{q}_{s_{1}}^{0} \bar{p}_{\ell s_{0}}=\bar{q}_{s_{1}}^{0} p_{\ell s_{0}} .
\end{aligned}
$$


Since $p_{\ell s_{1}}^{0}=\bar{p}_{\ell s_{1}}^{0}$, the above inequalities yield $q_{s_{1}}^{0}=\bar{q}_{s_{1}}^{0}$. We also have the equalities

$$
\begin{aligned}
& p_{s_{1}}^{0}=q_{s_{1}}^{0} p_{s_{1}}, \\
& \bar{p}_{s_{1}}^{0}=\bar{q}_{s_{1}}^{0} \bar{p}_{s_{1}}=q_{s_{1}}^{0} \bar{p}_{s_{1}} .
\end{aligned}
$$

Since $p_{s_{1}}^{0}=\bar{p}_{s_{1}}^{0}$, we find that $p_{s_{1}}=\bar{p}_{s_{1}}$.

Assume, for some $t \in\{1, \ldots, T-1\}$, we have shown that $p_{s_{t}}=\bar{p}_{s_{t}}$ and $q_{s_{t}}=\bar{q}_{s_{t}}$ for all $s_{t} \in S_{t}$. We complete the proof by showing that $p_{s_{t+1}}=\bar{p}_{s_{t+1}}$ and $q_{s_{t+1}}^{0}=\bar{q}_{s_{t+1}}^{0}$ for all $s_{t+1} \in S_{t+1}$.

Consider some $s_{t+1} \in S_{t+1}$ and let $\ell \in L$ be such that $s_{t+1} \in N_{\ell}^{\mathrm{s}}$. We have that

$$
\begin{aligned}
& p_{\ell s_{t+1}}^{0}=q_{s_{t+1}}^{0} p_{\ell s_{t+1}}=q_{s_{t+1}}^{0} p_{s_{t}\left(s_{t+1}\right)}=q_{s_{t+1}} q_{s_{t}\left(s_{t+1}\right)}^{0} p_{\ell s_{t}\left(s_{t+1}\right)}, \\
& \bar{p}_{\ell_{s_{t+1}}}^{0}=\bar{q}_{s_{t+1}}^{0} \bar{p}_{\ell s_{t+1}}=\bar{q}_{s_{t+1}}^{0} \bar{p}_{\ell s_{t}\left(s_{t+1}\right)}=\bar{q}_{s_{t+1}} \bar{q}_{s_{t}\left(s_{t+1}\right)}^{0} \bar{p}_{\ell s_{t}\left(s_{t+1}\right)}=\bar{q}_{s_{t+1}} q_{s_{t}\left(s_{t+1}\right)}^{0} p_{\ell s_{t}\left(s_{t+1}\right)} .
\end{aligned}
$$

Since $p_{\ell_{s t+1}}^{0}=\bar{p}_{\ell_{s_{t+1}}}^{0}$, the above inequalities yield $q_{s_{t+1}}=\bar{q}_{s_{t+1}}$, and since $q_{s_{t}\left(s_{t+1}\right)}^{0}=\bar{q}_{s_{t}\left(s_{t+1}\right)}^{0}$, we find that $q_{s_{t+1}}^{0}=\bar{q}_{s_{t+1}}^{0}$. We also have the equalities

$$
\begin{aligned}
& p_{s_{t+1}}^{0}=q_{s_{t+1}}^{0} p_{s_{t+1}}, \\
& \bar{p}_{s_{t+1}}^{0}=\bar{q}_{s_{t+1}}^{0} \bar{p}_{s_{t+1}}=q_{s_{t+1}}^{0} \bar{p}_{s_{t+1}} .
\end{aligned}
$$

Since $p_{s_{t+1}}^{0}=\bar{p}_{s_{t+1}}^{0}$, we find that $p_{s_{t+1}}=\bar{p}_{s_{t+1}}$, which completes the induction step.

It follows that $\alpha=p_{L^{*}}=\bar{p}_{L^{*}}=\bar{\alpha}$, which completes the proof.

\section{Extensions}

In a framework like ours, with multiple commodities at each date-event, there is no unique way to define price levels or inflation. We have taken the easiest definition for the price level, which defines the price level at a date-event to be equal to the price of an arbitrarily chosen commodity with a flexible price. A modest generalization would be to go from price levels to "activity" levels, which would naturally be parametrized by the variable $\rho$. In this case, the arbitrarily chosen commodity might be one with a sticky price, in which case the activity level would correspond to the amount of supply rationing or demand rationing. This seemingly modest extension of Theorem 6.1 and Corollary 6.2 does not hold.

Consider for the sake of simplicity an economy with two periods, zero nominal interest rates, a single date-event in period 1, and one commodity per date-event. Assume that the price in period 0 is flexible and the price in period 1 is sticky. Stickiness of the price in period 1 coupled with a zero nominal interest rate, implies that the present-value price of the future commodity is equal to the price of the current commodity. To show the existence of a sticky price equilibrium with $\rho_{s_{1}}=1 / 2$, we have to show existence of a sticky price 
equilibrium with no rationing in the future, and since there is a flexible price in period 0 , no rationing in the present. We therefore have to show the existence of an equilibrium in the Arrow-Debreu model where the prices of the two available commodities are equal to each other. Generically, such an equilibrium does not exist, which proves that Theorem 6.1 and Corollary 6.2 cannot be extended in this way. Still, there is an $\left|S_{1}\right|$-dimensional set of equilibria in this example, with a fixed amount of either supply or demand rationing in the market of the commodity at date-event $s_{1}$, parametrized by the price of the commodity at date-event $s_{0}$.

Rather than choosing a commodity with a flexible price for each terminal date-event, we might choose commodities with a flexible price at intermediate date-events. Such an extension can be proven by the same approach as in the proof of Theorem 6.1, with the obvious modifications.

Rather than choosing a single commodity at terminal date-events, one may define a price level at a terminal date-event, for instance by taking some weighted sum of the prices at that terminal date-event. It follows immediately from Corollary 6.2 that there are sticky price equilibria with arbitrarily high price levels at each terminal date-event.

The interest rate policy by the bank can depend on any exogenous shock as it is an arbitrary function of date-events in $S$. Since the date-events in $S$ need not be restricted to payoff relevant shocks, the interest rate policy could even depend on sunspots or be random. Our current approach, however, does not allow the interest rate to depend on past endogenous variables. Many papers in the macroeconomic literature have stressed the importance of such interest rate rules, see Woodford (2003) for a detailed discussion of this literature. Since interest rate rules are in general not compatible with the assumption that interest rates are restricted to some compact set, such an extension poses challenging equilibrium existence issues as it is not straightforward how the endogenous variables of the economy should be compactified. Although the equilibrium existence problem is challenging with interest rate rules, there is no reason to expect that the $\left|S_{T}\right|$-dimensional multiplicity of equilibrium will be lost as a result. The reason is that the imposition of $|S|$ interest rate rules will lead to $|S|$ no-arbitrage conditions, exactly the same as the number of conditions following from our approach with exogenous policies by the bank.

\section{Conclusion}

We have presented a general equilibrium level that has the same level of generality as the Arrow-Debreu model and that incorporates the main desiderata of the macroeconomic literature known as the new neoclassical synthesis. Agents form rational expectations on prices of commodities and assets, interest rates, supply constraints and demand constraints, 
in a stochastically developing multi-period economy. Commodity prices are allowed to be sticky, implying that monetary policy has non-trivial real consequences. At date-events where a commodity price is flexible, it does not adjust mechanically to some measure of disequilibrium, but is set at a market clearing level corresponding to the forces of supply and demand. Since price stickiness involves nominal prices, the model contains a general formulation of the monetary transaction technology.

The main result of the paper is that rational expectations are compatible with an $\left|S_{T}\right|$ dimensional set of equilibria and that in the presence of price stickiness this indeterminacy of equilibrium is real rather than nominal. This poses serious challenges to the issue of how households coordinate their expectations on one particular equilibrium and if they succeed in coordinating their expectations, on which equilibrium that will be. Under strong stationary assumptions and with flexible prices, it might seem natural that they coordinate on an equilibrium where future inflation is deterministic. Without stationary assumptions, and in the presence of price stickiness, such equilibria do generally not exist, and the issue of equilibrium selection becomes even more prominent.

\section{References}

Arrow, K.J. (1953), "Le Rôle des Valeurs Boursières pour la Répartition la Meilleure des Risques," in Econométrie, Colloques Internationaux du Centre National de la Recherche Scientifique, 40, 41-47; English version: (1964) "The Role of Securities in the Optimal Allocation of Risk-Bearing," Review of Economic Studies, 31, 91-96.

Baumol, W.J. (1952), "The Transactions Demand for Cash: An Inventory Theoretic Approach," Quarterly Journal of Economics, 66, 545-556.

Calvo, G.A. (1983), "Staggered Prices in a Utility-maximizing Framework," Journal of Monetary Economics, 12, 383-398.

Citanna, A., H. Crès, J. Drèze, P.J.J. Herings, and A. Villanacci (2001), "Continua of Underemployment Equilibria Reflecting Coordination Failures, Also at Walrasian Prices," Journal of Mathematical Economics, 36, 169-200.

Clower, R.W. (1967), "A Reconsideration of the Microfoundations of Monetary Theory," Western Economic Journal, 6, 1-9.

Cochrane, J.H. (2011), "Determinacy and Identification with Taylor Rules," Journal of Political Economy, 119, 565-615.

Debreu, G. (1959), Theory of Value, Yale University Press, New Haven, Connecticut.

Dixit, A.K., And J.E. Stiglitz (1977), "Monopolistic Competition and Optimum Product Diversity," American Economic Review, 67, 297-308.

DrÈze, J.H. (1975), "Existence of an Exchange Equilibrium under Price Rigidities," International Economic Review, 16, 301-320. 
Drèze, J.H., And H.M. Polemarchakis (2001), "Monetary Equilibria," in G. Debreu, W. Neuefeind, and W. Trockel (eds.), Economics Essays: A Festschrift for Werner Hildenbrand, Springer-Verlag, Heidelberg, pp. 83-108.

Goodfriend, M., And R.G. King (1997), "The New Neoclassical Synthesis and the Role of Monetary Policy," in B.S. Bernanke and J. Rotemberg (eds.), NBER Macroeconomics Annual 1997, Volume 12, pp. 231-296.

Herings, P.J.J. (1996A), "Equilibrium Existence Results for Economies with Price Rigidities," Economic Theory, 7, 63-80.

Herings, P.J.J. (1996B), Static and Dynamic Aspects of General Disequilibrium Theory, Theory and Decision Library, Series C: Game Theory, Mathematical Programming and Operations Research, Kluwer Academic Publishers, Norwell, Massachusetts.

Hildenbrand, W. (1974), Core and Equilibria of a Large Economy, Princeton University Press, Princeton.

LAAN, G. VAN DER (1982), "Simplicial Approximation of Unemployment Equilibria," Journal of Mathematical Economics, 9, 83-97.

Lucas, JR., R.E., And N.L. Stokey (1987), "Money and Rates of Interest in a Cash-in-Advance Economy," Econometrica, 55, 491-513.

Magill, M., And M. Quinzi (2010), "Expectations of Inflation, the Term Structure of Interest Rates, and Monetary Policy," Working Paper, 1-46.

Nakajima, T., and H.M. Polemarchakis (2005), "Money and Prices under Uncertainty," Review of Economic Studies, 72, 223-246.

Nakamura, E., And J. Steinsson (2008), "Five Facts about Prices: A Reevaluation of Menu Cost Models," Quarterly Journal of Economics, 123, 1415-1464.

Nakamura, E., And J. Steinsson (2010), "Monetary Non-neutrality in a Multisector Menu Cost Model," Quarterly Journal of Economics, 125, 961-1013.

Roberts, J., And H. Sonnenschein (1977), "On the Foundations of the Theory of Monopolistic Competition," Econometrica, 45, 101-113.

Sargent, T., And N. Wallace (1975), "'Rational" Expectations, the Optimal Monetary Instrument and the Optimal Money Supply Rule," Journal of Political Economy, 83, 241-254.

Sheshinski, E., And Y. Weiss (1977), "Inflation and Costs of Price Adjustment," Review of Economic Studies, 44, 287-303.

Svensson, L.E.O. (1986), "Sticky Goods Prices, Flexible Asset Prices, Monopolistic Competition, and Monetary Policy," Review of Economic Studies, 53, 385-405.

TAYlor, J.B. (1980), "Aggregate Dynamics and Staggered Contracts," Journal of Political Economy, $88,1-23$.

Tobin, J. (1956), "The Interest-Elasticity of Transactions Demand for Cash," Review of Economics and Statistics, 38, 241-247.

Woodford, M. (2003), Interest 83 Prices, Foundations of a Theory of Monetary Policy, Princeton University Press, Princeton, New Jersey. 